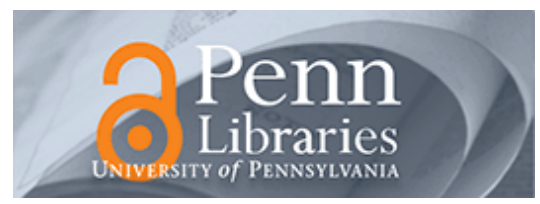

University of Pennsylvania

ScholarlyCommons

6-2012

\title{
A Dynamic Quality Ladder Model With Entry and Exit: Exploring the Equilibrium Correspondence Using the Homotopy Method
}

Ron N. Borkovsky

Ulrich Doraszelski

University of Pennsylvania

Yaroslav Kryukov

Follow this and additional works at: https://repository.upenn.edu/bepp_papers

Part of the Economics Commons, and the Marketing Commons

\section{Recommended Citation}

Borkovsky, R. N., Doraszelski, U., \& Kryukov, Y. (2012). A Dynamic Quality Ladder Model With Entry and Exit: Exploring the Equilibrium Correspondence Using the Homotopy Method. Quantitative Marketing and Economics, 10 (2), 197-229. http://dx.doi.org/10.1007/s11129-011-9113-4 


\title{
A Dynamic Quality Ladder Model With Entry and Exit: Exploring the Equilibrium Correspondence Using the Homotopy Method
}

\begin{abstract}
This paper explores the equilibrium correspondence of a dynamic quality ladder model with entry and exit using the homotopy method. This method is ideally suited for systematically investigating the economic phenomena that arise as one moves through the parameter space and is especially useful in games that have multiple equilibria. We briefly discuss the theory of the homotopy method and its application to dynamic stochastic games. We then present three main findings: First, the more costly and/or less beneficial it is to achieve or maintain a given quality level, the more a leader invests in striving to induce the follower to give up; the more quickly the follower does so; and the more asymmetric is the industry structure that arises. Second, the possibility of entry and exit gives rise to predatory and limit investment. Third, we illustrate and discuss the multiple equilibria that arise in the quality ladder model, highlighting the presence of entry and exit as a source of multiplicity.
\end{abstract}

\section{Keywords}

quality ladder model, dynamic oligopoly, homotopy method

Disciplines

Economics | Marketing 


\title{
A Dynamic Quality Ladder Model with Entry and Exit: Exploring the Equilibrium Correspondence Using the Homotopy Method*
}

\author{
Ron N. Borkovsky ${ }^{\dagger} \quad$ Ulrich Doraszelski $^{\ddagger} \quad$ Yaroslav Kryukov $^{\S}$
}

October 3, 2009

\begin{abstract}
This paper explores the equilibrium correspondence of a dynamic quality ladder model with entry and exit using the homotopy method. The homotopy method facilitates exploring the equilibrium correspondence in a systematic fashion; it is ideally suited for investigating the economic phenomena that arise as one moves through the paramter space and is especially useful in games that have multiple equilibria. We discuss the theory of the homotopy method and its application to dynamic stochastic games. We then present the following results: First, we find that a change in parameterization that increases (decreases) the cost (benefit) of achieving or maintaining any given product quality yields more asymmetric industry structures. Second, we show that the possibility of entry and exit alone gives rise to predatory and limit investment. Third, we illustrate and discuss the multiple equilibria that arise in the quality ladder model, highlighting the presence of entry and exit as a source of multiplicity.
\end{abstract}

\footnotetext{
${ }^{*}$ We are greatly indebted to Mark Satterthwaite for comments and suggestions. Borkovsky and Kryukov thank the General Motors Center for Strategy in Management at Northwestern's Kellogg School of Management for support during this project. Doraszelski gratefully acknowledges financial support from the National Science Foundation under Grant No. 0615615.

${ }^{\dagger}$ Rotman School of Management, University of Toronto, Toronto, ON M5S 3E6, ron.borkovsky@rotman.utoronto.ca.

${ }^{\ddagger}$ Department of Economics, Harvard University, Cambridge, MA 02138, doraszelski@harvard.edu.

$\S$ Tepper School of Business, Carnegie Mellon University, Pittsburgh, PA 15213, kryukov@cmu.edu.
} 


\section{Introduction}

Pakes \& McGuire (1994) develop a dynamic quality ladder model in the Markov perfect equilibrium framework of Ericson \& Pakes (1995). In the Pakes \& McGuire (1994) model, forward-looking oligopolistic firms compete with each other in the product market and through their investment, entry, and exit decisions. By investing in the present a firm hopes to increase the quality of its product-and ultimately its profits from product market competition-in the future. Investment, entry, and exit decisions are thus both dynamic and strategic.

The Pakes \& McGuire (1994) model has been widely used as a template for dynamic models of investment in the Ericson \& Pakes (1995) framework. It has been adapted to study mergers (Gowrisankaran 1999, Gowrisankaran \& Holmes 2004); capacity accumulation (Besanko \& Doraszelski 2004, Besanko, Doraszelski, Lu \& Satterthwaite 2008); competitive convergence (Langohr 2004); advertising (Doraszelski \& Markovich 2007, Dubé, Hitsch \& Manchanda 2005); network effects (Markovich 2008, Markovich \& Moenius 2009, Chen, Doraszelski \& Harrington 2009); research joint ventures (Song 2008); durable goods (Goettler \& Gordon 2009); investment in both vertical and horizontal product differentiation (Narajabad \& Watson 2008); spillovers (Laincz \& Rodrigues 2008); and the timing of version releases (Borkovsky 2008). The Pakes \& McGuire (1994) model has also been used to benchmark algorithms for computing Markov perfect equilibria in the Ericson \& Pakes (1995) framework 1

Although widely used and adapted, the Pakes \& McGuire (1994) model has never been thoroughly investigated. First, Pakes \& McGuire (1994) compute equilibria for just two parameterizations, thus leaving the parameter space largely unexplored. Second, Pakes \& McGuire (1994) do not characterize the equilibrium behavior that arises and instead focus on the effects of different institutional arrangements on market structure and welfare. Given the model's prominence, we feel it is important to better understand possible equilibrium behaviors and how behavior changes as one moves through the parameter space.

In this paper we use the homotopy method to undertake a thorough exploration of the equilibrium correspondence of a version of the Pakes \& McGuire (1994) model with at most two firms. The homotopy method was first applied to dynamic stochastic games by Besanko, Doraszelski, Kryukov \& Satterthwaite (2009) (see also Borkovsky et al. 2008). It is a type of path-following method. Starting from a single equilibrium that has already been computed, it traces out an entire path in the equilibrium correspondence by varying one or more selected parameters of the model. The homotopy method is thus ideally suited to investigating the economic phenomena that arise as one moves through the parameter space.

\footnotetext{
${ }^{1}$ See Pakes \& McGuire (1994), Pakes \& McGuire (2001), Ferris, Judd \& Schmedders (2007), Doraszelski \& Judd (2008), Weintraub, Benkard \& Van Roy (2008), Borkovsky, Doraszelski \& Kryukov (2008), Farias, Saure \& Weintraub (2008), and Santos (2009).
} 
We find that a change in parameterization that increases (decreases) the cost (benefit) of achieving/maintaining any given product quality yields more asymmetric industry structures in the short and long run. The cost is tied to the rate of depreciation and the effectiveness of investment, and the benefit is tied to the market size and the marginal cost of production. Consider an increase in the rate of depreciation: A higher rate of depreciation makes it more costly for a firm to achieve or maintain any given quality level for its product. It thus makes it more costly for the follower to catch up with the leader and thus stiffles the follower's incentive to invest. Accordingly, the leadership position becomes more secure. It follows that each firm strives to be the first to gain a lead over its rival and, thereafter, to induce its rival to cease investing and perhaps even exit, so that it can ultimately achieve industry dominance.

In a recent paper, Snider (2008) studies predation in the airline industry by structurally estimating a model of capacity accumulation similar to Besanko et al. (2008). He argues that cost asymmetries amongst firms give rise to predatory investment. We find that the possibility of entry and exit in the Pakes \& McGuire (1994) model alone gives rise to predatory and limit investment. This finding suggests that such behaviors are quite pervasive in the Ericson \& Pakes (1995) framework, especially since the Pakes \& McGuire (1994) model is arguably the simplest model in this framework that one can devise. Interestingly, we see predation occur in a complete information setting amongst symmetric firms whereas in much of the earlier literature (e.g., Milgrom \& Roberts 1982, Fudenberg \& Tirole 1986) predation occurs only in the face of asymmetric information and/or amongst asymmetric firms.

A second and equally important advantage of the homotopy method is that it allows us to systematically search for multiple equilibria. Multiple equilibria have long been a concern in the Ericson \& Pakes (1995) framework. They are problematic for at least two reasons. First, most structural estimation methods for models in the Ericson \& Pakes (1995) framework such as Aguirregabiria \& Mira (2007), Bajari, Benkard \& Levin (2007), Pakes, Ostrovsky \& Berry (2007), and Pesendorfer \& Schmidt-Dengler (2008) depend on the assumption that the same equilibrium is being played in all geographic markets and/or time periods. While this assumption is trivially satisfied if the equilibrium is unique, it has real bite in the presence of multiplicity. Second, it is difficult to draw conclusions from policy experiments if there are multiple equilibria, as one cannot determine which of them arises after a change in policy. It is therefore important to more fully characterize the set of equilibria in order to bound the range of outcomes that may be produced by the change in policy.

To date, the Pakes \& McGuire (1994) algorithm has been used most often to solve for Markov perfect equilibria in the Ericson \& Pakes (1995) framework. Being a Gaussian method, it cannot be used to systematically search for multiple equilibria; one can only take the trial-and-error approach of starting the algorithm from different points in the hope that 
it converges to different equilibria. The Pakes \& McGuire (1994) algorithm also suffers from a more severe problem: Besanko et al. (2009) show that when there are multiple equilibria, the Pakes \& McGuire (1994) algorithm is unable to compute a substantial fraction of them. In other words, they show that these equilibria are not locally stable under the Pakes \& McGuire (1994) algorithm.

The homotopy method is an important step towards resolving these issues, as it allows us to systematically search for multiplicity and to compute equilibria that are unstable under the Pakes \& McGuire (1994) algorithm. Recall that the homotopy method traces out an entire path in the equilibrium correspondence by varying one or more selected parameters of the model. If this path bends back on itself, then the homotopy method has identified multiple equilibria. The homotopy method is guaranteed to find all equilibria on a path it traverses and, therefore, to find all multiple equilibria that arise in this manner. However, since multiple equilibria for a given parameterization do not necessarily lie on the same path, the homotopy method is not guaranteed to find all equilibria.

Our systematic search reveals several instances of multiple equilibria, in contrast to Pakes \& McGuire's (1994) conclusion that "[we have computed several of our examples ... from different initial conditions, and we have always converged to the same fixed point, so nonuniqueness does not seem to be a problem with the simple functional forms we are currently using" (p. 570). In a companion paper (Borkovsky et al. 2008), we have explored the equilibrium correspondence of the quality ladder model without entry and exit. Interestingly, in the current model multiple equilibria arise for parameterizations for which we did not find multiple equilibria in the model without entry and exit. This suggests that entry and exit can by themselves be a source of multiplicity in the Ericson \& Pakes (1995) framework.

The paper proceeds as follows. In section 2, we present the Pakes \& McGuire (1994) model. In section 3, we briefly discuss the theory of the homotopy method as well as HOMPACK90, a suite of Fortran90 routines developed by Watson, Sosonkina, Melville, Morgan \& Walker (1997) that implements this method. We then explain how we use HOMPACK90 to compute equilibria of the quality ladder model. Section 4 describes the different types of equilibrium behavior that can arise and the associated industry dynamics. In section 5, we show that entry and exit can give rise to predatory and limit investment. In section 6, we describe instances of multiple equilibria that we have uncovered. Section 7 concludes.

\section{Quality Ladder Model}

We consider the quality ladder model of Pakes \& McGuire (1994). The description of the model is abridged; please see Pakes \& McGuire (1994) for details. To simplify the exposition, we restrict attention to a version of the model with at most two firms. To allow for entry and exit in a way that guarantees the existence of an equilibrium, we follow Doraszelski \& 
Satterthwaite (2009) and assume that setup costs and scrap values are privately observed random variables.

Firms and states. Firm $n \in\{1,2\}$ is described by its state $\omega_{n} \in\{0,1, \ldots, M\}$. States $1, \ldots, M$ describe the product quality of a firm that is active in the product market, i.e., an incumbent firm, while state 0 identifies the firm as being inactive, i.e., a potential entrant. We model exit as a transition from state $\omega_{n} \neq 0$ to $\omega_{n}^{\prime}=0$ and entry as a transition from state $\omega_{n}=0$ to state $\omega_{n}^{\prime} \neq 0$. The vector of firms' states is $\omega=\left(\omega_{1}, \omega_{2}\right) \in\{0, \ldots, M\}^{2}$ and we use $\omega^{[2]}$ to denote the vector $\left(\omega_{2}, \omega_{1}\right)$ obtained by interchanging firms' states.

Timing. In each period the sequence of events is as follows:

1. Incumbent firms learn their scrap value and decide on exit and investment. Potential entrants learn their setup cost and decide on entry.

2. Incumbent firms compete in the product market.

3. Exit and entry decisions are implemented.

4. The investment decisions of the remaining incumbents are carried out and their uncertain outcomes are realized. A common industry-wide depreciation shock affecting incumbents and entrants is realized.

Below we first describe the static model of product market competition and then turn to investment, entry, and exit dynamics.

Product market competition. The product market is characterized by price competition with vertically differentiated products. There is a continuum of consumers. Each consumer purchases at most one unit of one product. The utility a consumer derives from purchasing from firm $n$ is $g\left(\omega_{n}\right)-p_{n}+\epsilon_{n}$, where

$$
g\left(\omega_{n}\right)=\left\{\begin{array}{ccc}
-\infty & \text { if } & \omega_{n}=0, \\
\omega_{n} & \text { if } & 1 \leq \omega_{n} \leq \omega^{*}, \\
\omega^{*}+\ln \left(2-\exp \left(\omega^{*}-\omega_{n}\right)\right) & \text { if } & \omega^{*}<\omega_{n} \leq M,
\end{array}\right.
$$

maps the quality of the product into the consumer's valuation of it, $p_{n}$ is the price, and $\epsilon_{n}$ represents the consumer's idiosyncratic preference for product $n$. By setting $g(0)=-\infty$, we ensure that potential entrants have zero demand and thus do not compete in the product market. There is an outside alternative, product 0 , which has utility $\epsilon_{0}$. Assuming that the idiosyncratic preferences $\left(\epsilon_{0}, \epsilon_{1}, \epsilon_{2}\right)$ are independently and identically type 1 extreme value distributed, the demand for incumbent firm $n$ 's product is

$$
D_{n}(\mathbf{p} ; \omega)=m \frac{\exp \left(g\left(\omega_{n}\right)-p_{n}\right)}{1+\sum_{j=1}^{2} \exp \left(g\left(\omega_{j}\right)-p_{j}\right)},
$$


where $\mathbf{p}=\left(p_{1}, p_{2}\right)$ is the vector of prices and $m>0$ is the size of the market (the measure of consumers).

Incumbent firm $n$ chooses the price $p_{n}$ of its product to maximize profits. Hence, incumbent firm $n$ 's profits in state $\omega$ are

$$
\pi_{n}(\omega)=\max _{p_{n}} D_{n}\left(p_{n}, p_{-n}(\omega) ; \omega\right)\left(p_{n}-c\right),
$$

where $p_{-n}(\omega)$ is the price charged by the rival and $c \geq 0$ is the marginal cost of production. Given a state $\omega$, there exists a unique Nash equilibrium of the product market game (Caplin \& Nalebuff 1991). It is found easily by numerically solving the system of firstorder conditions corresponding to incumbent firms' profit-maximization problems. Note that product market competition does not directly affect state-to-state transitions. Hence, $\pi_{n}(\omega)$ can be computed before the Markov perfect equilibria of the dynamic stochastic game are computed. This allows us to treat $\pi_{n}(\omega)$ as a primitive in what follows.

Incumbent firms. Suppose first that firm $n$ is an incumbent firm, i.e., $\omega_{n} \neq 0$. We assume that at the beginning of each period each incumbent firm draws a random scrap value from a distribution $F(\cdot)$; in particular; we assume that scrap values are drawn from a triangular distribution with support $[\bar{\phi}-\epsilon, \bar{\phi}+\epsilon]$. Scrap values are independently and identically distributed across firms and periods. Incumbent firm $n$ learns its scrap value $\phi_{n}$ prior to making its exit and investment decisions, but the scrap values of its rivals remain unknown to it. If the scrap value is above a threshold $\tilde{\phi}_{n}$, then incumbent firm $n$ exits the industry and perishes; otherwise it remains in the industry. This decision rule can be represented either with the cutoff scrap value $\tilde{\phi}_{n}$ itself or with the probability $\xi_{n} \in[0,1]$ that incumbent firm $n$ remains in the industry in state $\omega$ because $\xi_{n}=\int 1\left(\phi_{n} \leq \tilde{\phi}_{n}\right) d F\left(\phi_{n}\right)=$ $F\left(\tilde{\phi}_{n}\right)$, where $1(\cdot)$ is the indicator function, is equivalent to $\tilde{\phi}_{n}=F^{-1}\left(\xi_{n}\right)$.

If it remains in the industry, then the state of an incumbent firm in the next period is determined by the stochastic outcomes of its investment decision and an industry-wide depreciation shock which stems from an increase in the quality of the outside alternative. In particular, incumbent firm $n$ 's state evolves according to the law of motion

$$
\omega_{n}^{\prime}=\omega_{n}+\tau_{n}-\eta
$$

where $\tau_{n} \in\{0,1\}$ is a random variable governed by incumbent firm $n$ 's investment $x_{n} \geq 0$ and $\eta \in\{0,1\}$ is an industry-wide depreciation shock. If $\tau_{n}=1$, the investment is successful and the quality of incumbent firm $n$ increases by one level. The probability of success is $\frac{\alpha x_{n}}{1+\alpha x_{n}}$, where $\alpha>0$ is a measure of the effectiveness of investment. If $\eta=1$, the industry is hit by a depreciation shock and the qualities of all products decrease by one level; this happens with probability $\delta \in[0,1]$. 
Potential entrants. Suppose next that firm $n$ is a potential entrant, i.e., $\omega_{n}=0$. We assume that at the beginning of each period each potential entrant draws a random setup cost from a distribution $F^{e}(\cdot)$; in particular, we assume that setup costs are drawn from a triangular distribution with support $\left[\bar{\phi}^{e}-\epsilon, \bar{\phi}^{e}+\epsilon\right]$. Like scrap values, setup costs are independently and identically distributed across firms and periods, and its setup cost is private to a firm. If the setup cost is below a threshold $\tilde{\phi}_{n}^{e}$, then potential entrant $n$ enters the industry; otherwise it perishes. This decision rule can be represented with the probability $\xi_{n} \in[0,1]$ that potential entrant $n$ enters in the industry.

Upon entry, a potential entrant undergoes a setup period. At the end of this period (i.e., at the beginning at the next period) potential entrant $n$ becomes incumbent firm $n$ and its state is

$$
\omega_{n}^{\prime}=\omega^{e}-\eta
$$

where $\omega^{e}$ is an exogenously given initial product quality.

Value and policy functions. Define $V_{n}(\omega)$ to be the expected net present value of firm $n$ 's cash flows if the industry is currently in state $\omega$. The incumbent's value function is $\mathbf{V}_{n}$ : $\{1, \ldots, M\} \times\{0, \ldots, M\} \rightarrow \mathbb{R}$, and its policy functions $\xi_{n}:\{1, \ldots, M\} \times\{0, \ldots, M\} \rightarrow[0,1]$ and $\mathbf{x}_{n}:\{1, \ldots, M\} \times\{0, \ldots, M\} \rightarrow[0, \infty)$ specify the probability that incumbent firm $n$ remains in the industry and its investment in state $\omega$. The potential entrant's value function is $\mathbf{V}_{n}:\{0\} \times\{0, \ldots, M\} \rightarrow \mathbb{R}$, and its policy function $\xi_{n}:\{0\} \times\{0, \ldots, M\} \rightarrow[0,1]$ specifies the probability that potential entrant $n$ enters the industry in state $\omega 2$

Bellman equation and optimality conditions. Suppose first that firm $n$ is an incumbent firm, i.e., $\omega_{n} \neq 0$. The value function $\mathbf{V}_{n}:\{1, \ldots, M\} \times\{0, \ldots, M\} \rightarrow \mathbb{R}$ is implicitly defined by the Bellman equation

$$
\begin{aligned}
V_{n}(\omega)= & \max _{\xi_{n} \in[0,1], x_{n} \geq 0} \pi_{n}(\omega)+\left(1-\xi_{n}\right) \mathrm{E}\left\{\phi_{n} \mid \phi_{n} \geq F^{-1}\left(\xi_{n}\right)\right\} \\
& +\xi_{n}\left\{-x_{n}+\beta\left(\frac{\alpha x_{n}}{1+\alpha x_{n}} W_{n}^{1}(\omega)+\frac{1}{1+\alpha x_{n}} W_{n}^{0}(\omega)\right)\right\},
\end{aligned}
$$

where $\beta \in(0,1)$ is the discount factor. Note that an optimizing incumbent cares about the expectation of the scrap value conditional on collecting it,

\footnotetext{
${ }^{2}$ We need not solve for the potential entrant's value function because it does not enter any of the equations below, aside from the one in which it is defined. This is because an incumbent firm that exits perishes; i.e., it does not become a potential entrant. We include the potential entrant's value function simply for the sake of completeness. Also recall that an entrant does not invest in the period in which it enters.
} 


$$
\mathrm{E}\left\{\phi_{n} \mid \phi_{n} \geq F^{-1}\left(\xi_{n}\right)\right\}=\left\{\begin{array}{ccc}
\bar{\phi} & \text { if } & T_{n}=-1, \\
\bar{\phi}+\epsilon\left(\frac{1-3 T_{n}{ }^{2}-2 T_{n}{ }^{3}}{3\left(2-\left(1+T_{n}\right)^{2}\right)}\right) & \text { if } & -1<T_{n}<0, \\
\bar{\phi}+\epsilon\left(\frac{1-3 T_{n}^{2}+2 T_{n}{ }^{3}}{3\left(1-T_{n}{ }^{2}\right)}\right) & \text { if } & 0 \leq T_{n}<1, \\
\bar{\phi}+\epsilon & \text { if } & T_{n}=1,
\end{array}\right.
$$

where

$$
T_{n} \equiv \frac{1}{\epsilon}\left[F^{-1}\left(\xi_{n}\right)-\bar{\phi}\right] \in[0,1],
$$

rather than its unconditional expectation $\mathrm{E}\left(\phi_{n}\right) . W_{n}^{\tau_{n}}(\omega)$ is the expectation of incumbent firm $n$ 's value function conditional on an investment success $\left(\tau_{n}=1\right)$ and failure $\left(\tau_{n}=0\right)$, respectively, as given by

$$
\begin{aligned}
W_{n}^{\tau_{n}}(\omega)= & \sum_{\eta \in\{0,1\}} \delta^{\eta}(1-\delta)^{1-\eta}\left[1\left(\omega_{-n}=0\right) \xi_{-n}(\omega) V_{n}\left(\max \left\{\min \left\{\omega_{n}+\tau_{n}-\eta, M\right\}, 1\right\}, \omega^{e}-\eta\right)\right. \\
& +1\left(\omega_{-n}>0\right)\left[\xi_{-n}(\omega) \sum_{\tau_{-n} \in\{0,1\}}\left(\frac{\alpha x_{-n}(\omega)}{1+\alpha x_{-n}(\omega)}\right)^{\tau_{-n}}\left(\frac{1}{1+\alpha x_{-n}(\omega)}\right)^{1-\tau_{-n}}\right. \\
& \left.\times V_{n}\left(\max \left\{\min \left\{\omega_{n}+\tau_{n}-\eta, M\right\}, 1\right\}, \max \left\{\min \left\{\omega_{-n}+\tau_{-n}-\eta, M\right\}, 1\right\}\right)\right] \\
& \left.+\left(1-\xi_{-n}(\omega)\right) V_{n}\left(\max \left\{\min \left\{\omega_{n}+\tau_{n}-\eta, M\right\}, 1\right\}, 0\right)\right],
\end{aligned}
$$

where $x_{-n}(\omega)$ is the investment of the rival in state $\omega$ and $\xi_{-n}(\omega)$ is the probability that a rival entrant (incumbent) enters (remains in) the industry in state $\omega$. Note that the min and max operators merely enforce the bounds of the state space.

Solving the maximization problem on the right-hand side of the Bellman equation (3) and using the fact that $\left(1-\xi_{n}\right) \mathrm{E}\left\{\phi_{n} \mid \phi_{n} \geq F^{-1}\left(\xi_{n}\right)\right\}=\int_{\phi_{n} \geq F^{-1}\left(\xi_{n}\right)} \phi_{n} d F\left(\phi_{n}\right)$, we obtain the first-order condition for $\xi_{n}(\omega)$ :

$$
-F^{-1}\left(\xi_{n}(\omega)\right)+\left\{-x_{n}+\beta\left(\frac{\alpha x_{n}}{1+\alpha x_{n}} W_{n}^{1}(\omega)+\frac{1}{1+\alpha x_{n}} W_{n}^{0}(\omega)\right)\right\}=0 .
$$

We further obtain the complementary slackness condition for $x_{n}(\omega)$ :

$$
\begin{aligned}
-1+\beta \frac{\alpha}{\left(1+\alpha x_{n}\right)^{2}}\left(W_{n}^{1}(\omega)-W_{n}^{0}(\omega)\right) & \leq 0 \\
x_{n}\left(-1+\beta \frac{\alpha}{\left(1+\alpha x_{n}\right)^{2}}\left(W_{n}^{1}(\omega)-W_{n}^{0}(\omega)\right)\right) & =0 \\
x_{n} & \geq 0 .
\end{aligned}
$$

Suppose next that firm $n$ is a potential entrant, i.e., $\omega_{n}=0$. The value function 
$\mathbf{V}_{n}:\{0\} \times\{0, \ldots, M\} \rightarrow \mathbb{R}$ is implicitly defined by

$$
V_{n}(\omega)=\max _{\xi_{n} \in[0,1]} \xi_{n}\left\{-\mathrm{E}\left\{\phi_{n}^{e} \mid \phi_{n}^{e} \leq F^{e-1}\left(\xi_{n}\right)\right\}+\beta W_{n}^{e}(\omega)\right\}
$$

Note that an optimizing potential entrant cares about the expectation of the setup cost conditional on entering,

$$
\mathrm{E}\left\{\phi_{n}^{e} \mid \phi_{n}^{e} \leq F^{e-1}\left(\xi_{n}\right)\right\}=\left\{\begin{array}{ccc}
\bar{\phi}-\epsilon & \text { if } & T_{n}^{e}=-1 \\
\bar{\phi}+\epsilon\left(\frac{-1+3 T_{n}^{e 2}+2 T_{n}^{e 3}}{3\left(\left(1+T_{n}^{e}\right)^{2}\right)}\right) & \text { if } & -1<T_{n}^{e}<0 \\
\bar{\phi}+\epsilon\left(\frac{-1+3 T_{n}^{e 2}-2 T_{n}^{e 3}}{3\left(2-\left(1-T_{n}^{e}\right)^{2}\right)}\right) & \text { if } & 0 \leq T_{n}^{e}<1 \\
\bar{\phi} & \text { if } & T_{n}^{e}=1
\end{array}\right.
$$

where

$$
T_{n}^{e} \equiv \frac{1}{\epsilon}\left[F^{e-1}\left(\xi_{n}\right)-\bar{\phi}\right]
$$

rather than its unconditional expectation $\mathrm{E}\left(\phi_{n}^{e}\right) . \quad W_{n}(\omega)$ is the expectation of potential entrant $n$ 's value function as given by

$$
\begin{aligned}
W_{n}^{e}(\omega)= & \sum_{\eta \in\{0,1\}} \delta^{\eta}(1-\delta)^{1-\eta}\left[1\left(\omega_{-n}=0\right) \xi_{-n}(\omega) V_{n}\left(\omega^{e}-\eta, \omega^{e}-\eta\right)+\right. \\
& 1\left(\omega_{-n}>0\right)\left[\xi_{-n}(\omega) \sum_{\nu_{-n} \in\{0,1\}}\left(\frac{\alpha x_{-n}(\omega)}{1+\alpha x_{-n}(\omega)}\right)^{\nu_{-n}}\left(\frac{1}{1+\alpha x_{-n}(\omega)}\right)^{1-\nu_{-n}}\right. \\
& \left.\times V_{n}\left(\omega^{e}-\eta, \max \left\{\min \left\{\omega_{-n}+\nu_{-n}-\eta, M\right\}, 1\right\}\right)\right] \\
& \left.+\left(1-\xi_{n}(\omega)\right) V_{n}\left(\omega^{e}-\eta, 0\right)\right]
\end{aligned}
$$

Using the fact that $-\xi_{n} \mathrm{E}\left\{\phi_{n}^{e} \mid \phi_{n}^{e} \leq F^{e-1}\left(\xi_{n}\right)\right\}=-\int_{\phi_{n}^{e} \leq F^{e-1}\left(\xi_{n}\right)} \phi_{n}^{e} d F^{e}\left(\phi_{n}^{e}\right)$, we obtain the first-order condition for $\xi_{n}$ :

$$
-F^{-1}\left(\xi_{n}\right)+\beta W_{n}^{e}(\omega)=0
$$

Equilibrium. We restrict attention to symmetric Markov perfect equilibria in pure strategies. Proposition 3 in Doraszelski \& Satterthwaite (2009) establishes that such an equilibrium always exists. In a symmetric equilibrium, the investment decision taken by firm 2 in state $\omega$ is identical to the investment decision taken by firm 1 in state $\omega^{[2]}$, i.e., $x_{2}(\omega)=x_{1}\left(\omega^{[2]}\right)$, and similarly for the entry/exit decisions, and the value functions. It therefore suffices to determine the value and policy functions of firm 1, and we define $V(\omega)=V_{1}(\omega), \xi(\omega)=\xi_{1}(\omega)$, and $x(\omega)=x_{1}(\omega)$ for each state $\omega$. Similarly, we define $W^{\tau_{1}}(\omega)=W_{1}^{\tau_{1}}(\omega)$ and $W^{e}(\omega)=W_{1}^{e}(\omega)$ for each state $\omega$. Solving for an equilibrium for a particular parameterization of the model amounts to finding a value function $\mathbf{V}(\cdot)$ and pol- 
icy functions $\xi(\cdot)$ and $\mathbf{x}(\cdot)$ that satisfy the Bellman equations (3) and (7) and the optimality conditions (5), (6), and (9).

\section{Computation}

Our objective is to compute equilibria of the model using the homotopy method. In subsection 3.1, we present the theory of the homotopy method. In subection 3.2, we discuss HOMPACK90, a suite of Fortran90 routines developed by Watson et al. (1997) that implements this method. In subsection 3.3, we explain how we apply this method to the quality ladder model.

\subsection{The Homotopy Method}

The homotopy method attempts to describe the equilibrium correspondence that maps model parameters into equilibria in a tractable manner. First, it represents the system of nonlinear equations that characterizes the equilibrium correspondence as a collection of smooth paths 3 Second, it characterizes these paths using a system of ordinary differential equations. An implementation of the method - a homotopy algorithm - can be used to trace out the paths by numerically solving this system. As such, a homotopy algorithm can be used to explore an equilibrium correspondence in a systematic fashion; starting from a single equilibrium that has already been computed for a given parameterization of the model, a homotopy algorithm traces out an entire path of equilibria by varying a parameter of interest. It is therefore especially useful in searching for multiple equilibria.

The equilibrium conditions depend on the parameterization of the model. Making this dependence explicit, the equilibrium conditions can be written as

$$
\mathbf{H}(\mathbf{z}, \lambda)=\mathbf{0},
$$

where $\mathbf{H}: \mathbb{R}^{N+1} \rightarrow \mathbb{R}^{N}, \mathbf{z} \in \mathbb{R}^{N}$ is the vector of the unknown values and policies, $\mathbf{0} \in \mathbb{R}^{N}$ is a vector of zeros, and $\lambda \in[0,1]$ is the so-called homotopy parameter. We use boldface to distinguish between vectors and scalars. Depending on the application at hand, the homotopy parameter maps into one or more of the parameters of the model. The object of interest in the equilibrium correspondence

$$
\mathbf{H}^{-1}=\{(\mathbf{z}, \lambda) \mid \mathbf{H}(\mathbf{z}, \lambda)=\mathbf{0}\} .
$$

A homotopy algorithm aims to trace out entire paths of equilibria in $\mathbf{H}^{-1}$. This allows us to understand how equilibrium behavior changes as we move through the parameter space.

\footnotetext{
${ }^{3}$ In subsection 3.3 we explain how to formulate the equilibrium conditions as a system of equations.
} 


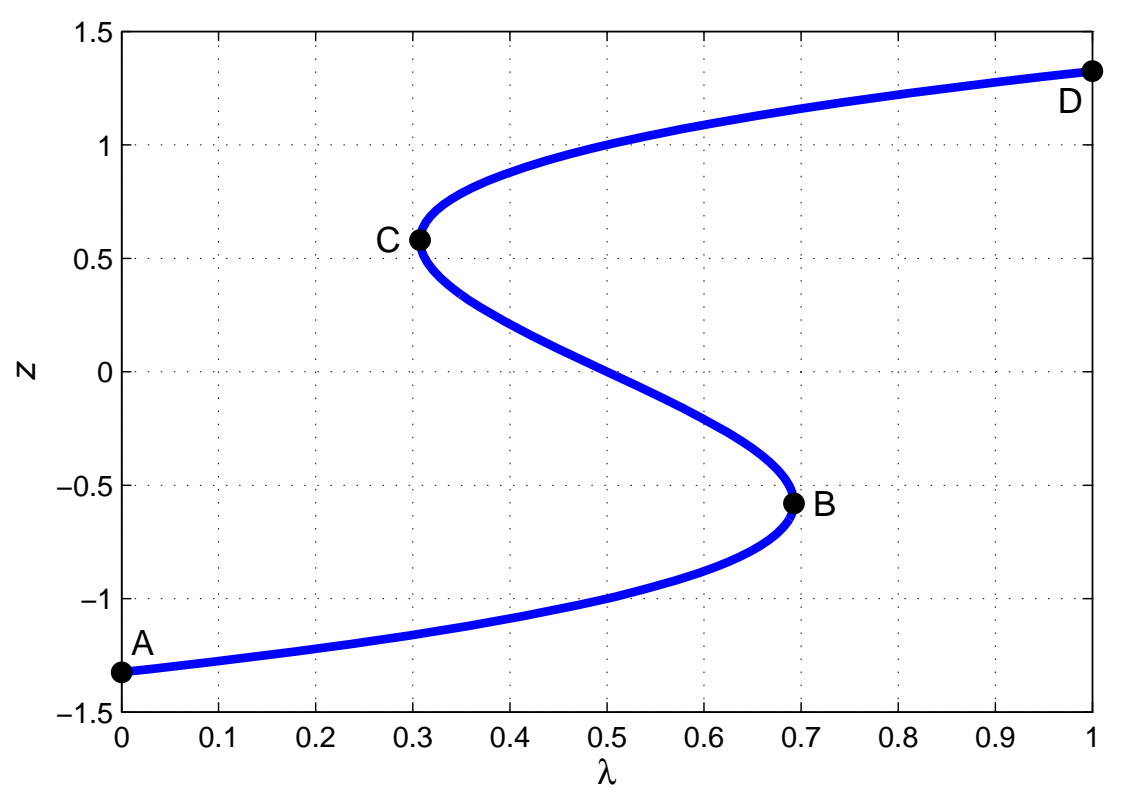

Figure 1: Example.

Example. We use a simple example to explain how the homotopy method describes the solution set. Let $N=1$ and consider the equation $H(z, \lambda)=0$ that relates a variable $z$ with a parameter $\lambda$, where

$$
H(z, \lambda)=z^{3}-z+1-2 \lambda .
$$

Here we do not use boldface for $z$ and 0 since they are scalars. The set of solutions is $H^{-1}=\{(z, \lambda) \mid H(z, \lambda)=0\}$ and is shown in Figure 1. Inspecting Figure 1, one can easily see that multiple solutions arise whenever the graph bends back on itself, as it does at points B and C. For example, at $\lambda=0.5$ there are three solutions, namely $z=-1, z=0$, and $z=1$. Thus the mapping from $\lambda$ to $z$ is a correspondence and cannot be described by a function.

The homotopy method constructs a parametric path $(z(s), \lambda(s)) \in H^{-1}$ through the set of solutions. The points on this path are indexed by the auxiliary variable $s$ that increases or decreases monotonically as we move along the path. To construct the parametric path, we proceed as follows. As $(z(s), \lambda(s)) \in H^{-1}$, it follows that $H(z(s), \lambda(s))=0$ for all $s$. Totally differentiating with respect to $s$ yields the condition for remaining on the path:

$$
\frac{\partial H(z(s), \lambda(s))}{\partial z} z^{\prime}(s)+\frac{\partial H(z(s), \lambda(s))}{\partial \lambda} \lambda^{\prime}(s)=0 .
$$

As this is one differential equation in two unknowns, $z^{\prime}(s)$ and $\lambda^{\prime}(s)$, it has many solutions; 
however, they all describe the same path in $H^{-1}$. One obvious solution is

$$
\begin{aligned}
z^{\prime}(s) & =\frac{\partial H(z(s), \lambda(s))}{\partial \lambda}=-2, \\
\lambda^{\prime}(s) & =-\frac{\partial H(z(s), \lambda(s))}{\partial z}=-3 z^{2}+1 .
\end{aligned}
$$

The so-called basic differential equations (BDE) (12) and (13) and the initial condition

$$
H(\gamma, 0)=0
$$

where

$$
\gamma=-\frac{(108+12 \sqrt{69})^{2 / 3}+12}{6(108+12 \sqrt{69})^{1 / 3}}
$$

describe the parametric path $(z(s), \lambda(s)) \in H^{-1}$ given by

$$
\begin{aligned}
& z(s)=-2 s+\gamma \\
& \lambda(s)=-3 s(-2 s+\gamma)^{2}+s .
\end{aligned}
$$

While this simple example allows for an analytic solution to the BDE, most real-world problems do not; therefore, numerical methods are typically used to solve the BDE.

A homotopy algorithm traces out the parametric path $(z(s), \lambda(s)) \in H^{-1}$ by computing a sequence of points on the path. The unique solution to $H(z, \lambda)=0$ when $\lambda=0$ is $z=\gamma$. This provides the homotopy algorithm with a starting point (point $A$ in Figure 1). From there the algorithm uses the basic differential equations (12) and (13) to determine the direction in which it should proceed to find the next point on the path. It continues in this manner until it reaches $\lambda=1$ (point $D$ ).

We can now proceed with the general case as we did in the example. Recall that the object of interest is the equilibrium correspondence $\mathbf{H}^{-1}=\{(\mathbf{z}, \lambda) \mid \mathbf{H}(\mathbf{z}, \lambda)=\mathbf{0}\}$. We define the parametric path $(\mathbf{z}(s), \lambda(s)) \in \mathbf{H}^{-1}$. Totally differentiating $\mathbf{H}(\mathbf{z}(s), \lambda(s))=\mathbf{0}$ with respect to $s$ yields

$$
\frac{\partial \mathbf{H}(\mathbf{z}(s), \lambda(s))}{\partial \mathbf{z}} \mathbf{z}^{\prime}(s)+\frac{\partial \mathbf{H}(\mathbf{z}(s), \lambda(s))}{\partial \lambda} \lambda^{\prime}(s)=\mathbf{0},
$$

where $\frac{\partial \mathbf{H}(\mathbf{z}(s), \lambda(s))}{\partial \mathbf{z}}$ is the $(N \times N)$ Jacobian of $\mathbf{H}$ with respect to $\mathbf{z}, \mathbf{z}^{\prime}(s)$ and $\frac{\partial \mathbf{H}(\mathbf{z}(s), \lambda(s))}{\partial \lambda}$ are $(N \times 1)$ vectors, and $\lambda^{\prime}(s)$ is a scalar. This is a system of $N$ differential equations in $N+1$ unknowns, $z_{i}^{\prime}(s), i=1, \ldots, N$, and $\lambda^{\prime}(s)$. Zangwill \& Garcia (1981) show that this system has a solution that satisfies the basic differential equations

$$
y_{i}^{\prime}(s)=(-1)^{i+1} \operatorname{det}\left(\left[\frac{\partial \mathbf{H}(\mathbf{y}(s))}{\partial \mathbf{y}}\right]_{-i}\right), \quad i=1, \ldots, N+1
$$


where $\mathbf{y}(s)=(\mathbf{z}(s), \lambda(s))$, and the notation $[\cdot]_{-i}$ is used to indicate that the $i$ th column is removed from the $(N \times(N+1))$ Jacobian $\frac{\partial \mathbf{H}(\mathbf{y}(s))}{\partial \mathbf{y}}$ of $\mathbf{H}$ with respect to $\mathbf{y}$ (see pp. 27-28). Note that equations (12) and (13) are the basic differential equations (18) for the special case of $N=1$.

Regularity and smoothness requirements. A closer inspection of the basic differential equations (18) reveals a potential difficulty. If the Jacobian $\frac{\partial \mathbf{H}(\mathbf{y}(s))}{\partial \mathbf{y}}$ is not of full rank at some point $\mathbf{y}(s)$ on the solution path, then the determinant of each of its square submatrices is zero. Thus, according to the basic differential equations (18), $y_{i}^{\prime}(s)=0, i=1, \ldots, N+1$; the fact that the system of differential equations is underdetermined presents a problem for a homotopy algorithm that exploits the basic differential equations to determine the direction in which to proceed, as they are uninformative at such a point. A central condition in the mathematical literature on the homotopy method is thus that the Jacobian must have full rank at all points on the solution path. If so, the homotopy is called regular. More formally, $\mathbf{H}$ is regular if $\operatorname{rank}\left(\frac{\partial \mathbf{H}(\mathbf{y})}{\partial \mathbf{y}}\right)=N$ for all $\mathbf{y} \in \mathbf{H}^{-1}$.

The other major requirement of the homotopy method is smoothness in the sense of differentiability. This yields solution paths that are smooth and free of sudden turns or kinks. Formally, if $\mathbf{H}$ is continuously differentiable in addition to regular, then the set of solutions $\mathbf{H}^{-1}$ consists only of continuously differentiable paths. This result is known as the path theorem and essentially follows from the implicit function theorem (see, e.g., p. 20 of Zangwill \& Garcia 1981). Moreover, for a path to be described by the basic differential equations (18) it must be the case that $\mathbf{H}$ is twice continuously differentiable in addition to regular. This result is known as the BDE theorem (see pp. 27-28 of Zangwill \& Garcia 1981).

If the regularity and smoothness requirements are satisfied, the solution set $\mathbf{H}^{-1}$ consists only of smooth paths that can be easily traversed by a homotopy algorithm. In particular, the solution set $\mathbf{H}^{-1}$ consists only of paths that start at $\lambda=0$ and end at $\lambda=1$; paths that start and end at $\lambda=0$ or $\lambda=1$; loops; and paths that start at $\lambda=0$ or $\lambda=1$ but never end because $z$ (or a component of $\mathbf{z}$ in the case of a vector) tends to $+\infty$ or $-\infty$. Regularity allows us to rule out isolated equilibria, pitchfork bifurcations, infinite spirals, and paths that suddenly terminate. See Section 2 of Borkovsky et al. (2008) for illustrative figures and further details.

In practice, it is often hard to establish regularity because the Jacobian of a system of equations that characterizes the equilibria of a dynamic stochastic game formulated in the Ericson \& Pakes (1995) framework tends to be intractable. This stems partly from the fact that the Jacobian for such a system is typically quite large because the system includes at least two equations (Bellman equation and optimality condition) for each state of the industry, and even "small" models with few firms and few states per firm tend to have hundreds of industry states. The smoothness requirement can often be satisfied by a judicious choice of functional forms. 
Recall that in Section 2 we assume that scrap values and setup costs are drawn from triangular distributions; the resulting cumulative distribution functions are once but not twice continuously differentiable, contrary to the smoothness requirement. We nevertheless did not encounter a problem. If a problem is encountered in another application, we suggest using a $\operatorname{Beta}(k, k)$ distribution with $k \geq 3$ to ensure that the system of equations is at least twice continuously differentiable.

\subsection{HOMPACK90 Software Package}

HOMPACK90 is a suite of Fortran90 routines that traces out a path in $\mathbf{H}^{-1} 4$ In order to use HOMPACK90, first, the user must provide Fortran90 code that returns $\mathbf{H}(\mathbf{z}, \lambda)$ at a given point $(\mathbf{z}, \lambda)$. Second, the user must provide a routine that returns the Jacobian of $\mathbf{H}$ at a given point $(\mathbf{z}, \lambda)$. Many applications yield Jacobians with relatively few nonzeros elements; such a Jacobian is called sparse and HOMPACK90 allows the user to store such a Jacobian using a sparse-matrix storage format. This can substantially decrease computation time; however, in order to use this format, the user must specify the "sparsity structure" of the Jacobian, i.e., the row and column indices of potentially non-zero elements. The Jacobian can be computed either numerically (see, e.g., Chapter 7 of Judd 1998) or analytically. We compute the Jacobian analytically using ADIFOR, a program that analytically differentiates Fortran code. ADIFOR is described in Bischof, Khademi, Mauer \& Carle (1996). Third, the user must provide an initial condition in the form of a solution to the system of equations for the particular parameterization associated with $\lambda=0$. In some cases, if the parameterization associated with $\lambda=0$ is trivial, the solution can be derived analytically. More generally, a solution for a particular parameterization can be computed numerically using a number of approaches such as Gaussian methods including (but not limited to) the Pakes \& McGuire (1994) algorithm, other nonlinear solvers (see Ferris et al. 2007), and artificial homotopies (see Borkovsky et al. 2008). Borkovsky et al. (2008) discuss the three inputs as well as a number of potential problems with HOMPACK90 in detail. A technical description of HOMPACK90 is given in Watson, Billups \& Morgan (1987) and Watson et al. (1997).

\subsection{Application to Quality Ladder Model}

As explained above, the homotopy method operates on a system of equations. However, given the non-negativity constraint on investment, the problem that an incumbent firm has to solve is formulated using a complementary slackness condition, a combination of

\footnotetext{
${ }^{4}$ There are other software packages that can be used to solve systems of equations using the homotopy method. For example, with the freely-available Gambit (McKelvey, McLennan \& Turocy 2006) and PHCpack (Verschelde 1999) software packages, one can use the homotopy method to obtain solutions to polynomial systems; these algorithms exploit the structure of a polynomial system, in particular its sparsity structure. We use HOMPACK90 because it does not impose any restrictions on the functional form of the system of equations or the sparsity structure of the Jacobian.
} 
equalities and inequalities, rather than a first-order condition, an equation. Fortunately, Zangwill \& Garcia (1981) offer a reformulation of the complementary slackness condition that consists entirely of equations that are continuously differentiable to an arbitrary degree (see pp. 65-68).

Consider the complementary slackness condition (66). Using the fact that we focus on symmetric equilibria in order to eliminate firm indices and multiplying through by $(1+$ $\alpha x(\omega))^{2}$ to simplify the expressions that arise in what follows, the complementary slackness condition (6) can be restated as

$$
\begin{aligned}
-(1+\alpha x(\omega))^{2}+\beta \alpha\left(W^{1}(\omega)-W^{0}(\omega)\right) & \leq 0, \\
x(\omega)\left(-\left(1+\alpha x(\omega)^{2}+\beta \alpha\left(W^{1}(\omega)-W^{0}(\omega)\right)\right)\right. & =0, \\
x(\omega) & \geq 0 .
\end{aligned}
$$

Now introduce another scalar variable $\zeta(\omega)$ and consider the system of equations

$$
\begin{aligned}
-(1+\alpha x(\omega))^{2}+\beta \alpha\left(W^{1}(\omega)-W^{0}(\omega)\right)+[\max \{0, \zeta(\omega)\}]^{k} & =0, \\
-x(\omega)+[\max \{0,-\zeta(\omega)\}]^{k} & =0
\end{aligned}
$$

where $k \in \mathbb{N}$. It is easy to see that the system of equations (20) and (21) is equivalent to the complementary slackness condition (19) 5 This system is $(k-1)$ times continuously differentiable with respect to $\zeta(\omega)$. Hence, by choosing $k$ large enough, we can satisfy the smoothness requirement of the homotopy method. The terms $[\max \{0, \zeta(\omega)\}]^{k}$ and $[\max \{0,-\zeta(\omega)\}]^{k}$ serve as slack variables that ensure that the inequalities in (19) are satisfied and the fact that $[\max \{0, \zeta(\omega)\}]^{k}[\max \{0,-\zeta(\omega)\}]^{k}=0$ ensures that the equality in (19) holds.

We could now proceed to define the system of homotopy equations using equations (201) and (21), the incumbent's Bellman equation in (3) and the first-order condition for $\xi(\omega)$ in (5) for $\omega \in\{1, \ldots, M\} \times\{0, \ldots, M\}$, and the entrant's Bellman equation in (7) and the first-order condition for $\xi(\omega)$ in (9) for $\omega \in\{0\} \times\{0, \ldots, M\} 6$ This would yield a system of $2(M+1)(2 M+1)$ equations in the $2(M+1)(2 M+1)$ unknowns $V(\omega)$ and $\xi(\omega)$ for $\omega \in\{0, \ldots, M\}^{2}$, and $x(\omega)$ and $\zeta(\omega)$ for $\omega \in\{1, \ldots, M\} \times\{0, \ldots, M\}$. However, two problems arise: First, because we have added the slack variables, this system of equations is relatively large with $2(M+1)(2 M+1)$ equations and unknowns. This leads to increased

\footnotetext{
${ }^{5}$ From equations (20) and (21) it follows that $\zeta(\omega)=\left\{\begin{array}{ccc}{\left[(1+\alpha x(\omega))^{2}+\beta \alpha\left(W^{1}(\omega)-W^{0}(\omega)\right)\right]^{1 / k}} & \text { if } & -(1+\alpha x(\omega))^{2}+\beta \alpha\left(W^{1}(\omega)-W^{0}(\omega)\right)<0, \\ -[x(\omega)]^{1 / k} & \text { if } & x(\omega)>0, \\ 0 & \text { if } & -(1+\alpha x(\omega))^{2}+\beta \alpha\left(W^{1}(\omega)-W^{0}(\omega)\right)=x(\omega)=0 .\end{array}\right.$ The claim now follows from the solution for $\zeta(\omega)$ in equation 22 and the fact $\max \{0,-\zeta(\omega)\} \max \{0, \zeta(\omega)\}=0$.

${ }^{6}$ To be precise, we would substitute the equilibrium entry/exit policy $\xi(\omega)$ for $\xi_{n}$ in (3) and (7), and the equilibrium investment policy $x(\omega)$ for $x_{n}$ in (3); accordingly, we would remove the max operators.
} 
memory requirements and computation time. Second, this system of equations yields an extremely sparse Jacobian, and we have found that this tends to cause HOMPACK90's sparse linear equation solver to fail; this is discussed further in Borkovsky et al. (2008).

We address these problems by solving equation (21) for $x(\omega)$,

$$
x(\omega)=[\max \{0,-\zeta(\omega)\}]^{k},
$$

and then substituting this into equations (3), (15), (17), (9), and (20). This reduces the system of $2(M+1)(2 M+1)$ equations in $2(M+1)(2 M+1)$ unknowns by $M(M+1)$ equations and unknowns, respectively, to a system of $(M+1)(3 M+2)$ equations in $(M+1)(3 M+2)$ unknowns. Moreover, it eliminates excessive sparsity.

To this end, define the vector of unknowns in equilibrium as

$$
\begin{aligned}
\mathbf{z}= & {[V(0,0), V(1,0), \ldots, V(M, 0), V(0,1), \ldots, V(M, M),} \\
& \xi(0,0), \ldots, \xi(M, M), \zeta(1,0), \ldots, \zeta(M, M)] .
\end{aligned}
$$

The equations comprise

$$
\begin{gathered}
H_{\omega}^{1}(\mathbf{z}, \lambda)=-V(\omega)+\pi_{1}(\omega)+(1-\xi(\omega)) \mathrm{E}\left\{\phi_{n} \mid \phi_{n} \geq F^{-1}(\xi(\omega))\right\} \\
+\xi(\omega)\left\{-x(\omega)+\beta\left(\frac{\alpha x(\omega)}{1+\alpha x(\omega)} W^{1}(\omega)+\frac{1}{1+\alpha x(\omega)} W^{0}(\omega)\right)\right\}=0 \\
H_{\omega}^{2}(\mathbf{z}, \lambda)=-F^{-1}(\xi(\omega))+\left\{-x(\omega)+\beta\left(\frac{\alpha x(\omega)}{1+\alpha x(\omega)} W^{1}(\omega)+\frac{1}{1+\alpha x(\omega)} W^{0}(\omega)\right)\right\}=0 \\
H_{\omega}^{3}(\mathbf{z}, \lambda)=-(1+\alpha x(\omega))^{2}+\beta \alpha\left(W^{1}(\omega)-W^{0}(\omega)\right)+[\max \{0, \zeta(\omega)\}]^{k}=0
\end{gathered}
$$

for states $\omega \in\{1, \ldots, M\} \times\{0, \ldots, M\}$, and

$$
\begin{gathered}
H_{\omega}^{1}(\mathbf{z}, \lambda)=-V(\omega)+\xi(\omega)\left\{-\mathrm{E}\left\{\phi_{1}^{e} \mid \phi_{1}^{e} \leq F^{e-1}(\xi(\omega))\right\}+\beta W^{e}(\omega)\right\}=0, \\
H_{\omega}^{2}(\mathbf{z}, \lambda)=-F^{-1}(\xi(\omega))+\beta W^{e}(\omega)=0
\end{gathered}
$$

for states $\omega \in\{0\} \times\{0, \ldots, M\}$, where we substitute for $W^{\tau_{1}}(\omega)$ using the definition in (44), for $W^{e}(\omega)$ using the definition in (8), and for $x(\omega)$ using (23). Note that (24), (25), (26), (27), and (28) are equations that are used to construct the system of homotopy equations, while (4), (8)), and (23) are simply definitional shorthands for terms that appear in the aforementioned equations. The collection of equations (24), (25), and (26) for states $\omega \in\{1, \ldots, M\} \times\{0, \ldots, M\}$, and (27) and (28) for states $\omega \in\{0\} \times\{0, \ldots, M\}$ can be written 


\begin{tabular}{r|ccccccccccc} 
parameter & $M$ & $m$ & $c$ & $\omega^{*}$ & $\beta$ & $\alpha$ & $\delta$ & $\bar{\phi}$ & $\bar{\phi}^{e}$ & $\epsilon$ & $\omega^{e}$ \\
\hline value & 18 & 5 & 5 & 12 & 0.925 & 3 & 0.7 & 3 & 1 & 1 & 4
\end{tabular}

Table 1: Parameter values.

more compactly as

$$
\mathbf{H}(\mathbf{z}, \lambda)=\left[\begin{array}{c}
H_{(0,0)}^{1}(\mathbf{z}, \lambda) \\
H_{(1,0)}^{1}(\mathbf{z}, \lambda) \\
\vdots \\
H_{(M, M)}^{3}(\mathbf{z}, \lambda)
\end{array}\right]=\mathbf{0}
$$

where $\mathbf{0} \in \mathbb{R}^{(M+1)(3 M+2)}$ is a vector of zeros. Any solution to this system of $(M+1)(3 M+2)$ equations in $(M+1)(3 M+2)$ unknowns, $\mathbf{z} \in \mathbb{R}^{(M+1)(3 M+2)}$, is a symmetric equilibrium in pure strategies (for a given value of $\lambda \in[0,1]$ ). The equilibrium investment decision $x(\omega)$ in state $\omega$ is recovered by substituting the equilibrium slack variable $\zeta(\omega)$ into definition (23).

Parameterization. The baseline parameterization is presented in Table 1 and is identical to the parameterization explored in Pakes \& McGuire (1994) except that we assume higher setup costs and scrap values than Pakes \& McGuire (1994). The reason is that we are interested in studying an industry that can support up to two active firms, while they study an industry that can support up to six active firms.

As explained above, the homotopy algorithm traces out an entire path of equilibria by varying one or more parameters of interest. We allow $\beta, \alpha, \delta, \bar{\phi}$ and $\bar{\phi}^{e}$ to vary, while holding the remaining parameters fixed at the baseline values. As such, we make the vector comprising $\beta, \alpha, \delta, \bar{\phi}$ and $\bar{\phi}^{e}$ a function of the homotopy parameter $\lambda$ :

$$
\left[\begin{array}{c}
\beta(\lambda) \\
\alpha(\lambda) \\
\delta(\lambda) \\
\bar{\phi}(\lambda) \\
\bar{\phi}^{e}(\lambda)
\end{array}\right]=\left[\begin{array}{l}
\beta^{\text {start }} \\
\alpha^{\text {start }} \\
\delta^{\text {start }} \\
\bar{\phi}^{\text {start }} \\
\bar{\phi}^{e^{\text {start }}}
\end{array}\right]+\lambda\left[\begin{array}{c}
\beta^{\text {end }}-\beta^{\text {start }} \\
\alpha^{\text {end }}-\alpha^{\text {start }} \\
\delta^{\text {end }}-\delta^{\text {start }} \\
\bar{\phi}^{\text {end }}-\bar{\phi}^{\text {start }} \\
\bar{\phi}^{\text {end }}-\bar{\phi}^{\text {start }}
\end{array}\right]
$$

For example, if $\delta^{\text {start }}=0$ and $\delta^{\text {end }}=1$ while $\beta^{\text {start }}=\beta^{\text {end }}, \alpha^{\text {start }}=\alpha^{\text {end }}, \bar{\phi}^{\text {start }}=\bar{\phi}^{\text {end }}$, and $\bar{\phi}^{e^{\text {start }}}=\bar{\phi}^{e^{e n d}}$, then the homotopy algorithm traces out the equilibrium correspondence from $\delta(0)=0$ to $\delta(1)=1$, holding all other parameter values fixed. Setting different starting and ending values for one or more of these parameters allows us to explore the set of equilibria by moving through the parameter space in various directions. In general, given any starting and ending values for the parameter vector, the homotopy algorithm can trace out an entire path of equilibria by moving along the line in parameter space that connects the starting and ending values. 


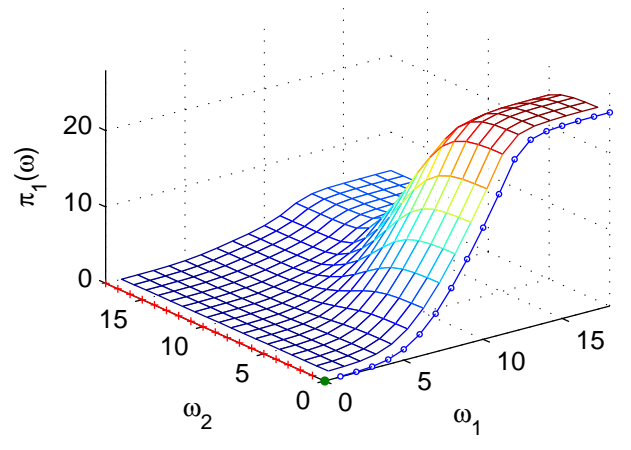

Figure 2: Profit function $\pi_{1}(\omega)$. (Blue $=$ incumbent monopolist; red $=$ potential entrant; green $=$ two potential entrants.)

Code. A set of code that allows the user to compute equilibria of the quality ladder model using the homotopy method is available on the authors' homepages. It includes (i) Matlab code that implements the Pakes \& McGuire (1994) algorithm that we use to compute a starting point for the homotopy algorithm; (ii) Fortran90 code that includes HOMPACK90 and the implementation of the quality ladder model; and (iii) additional Matlab code that analyzes the output of the homotopy algorithm. More detailed information is included within the code itself.

\section{Equilibrium Behavior and Industry Dynamics}

Equilibrium behavior is driven by the benefits and costs of product quality. The benefits of product quality stem from the product market; a higher product quality yields a higher market share and, accordingly, higher profits. We begin by examining firm 1's profit function in Figure 2 more closely; as firms are symmetric, firm 2 faces a symmetric profit function. The profit function of an incumbent monopolist is plotted in blue along the $\omega_{1}$ axis. The profit function of a potential entrant facing an incumbent monopolist is plotted in red along the $\omega_{2}$ axis. The profit of a potential entrant facing an empty industry is plotted in green over state $(0,0)$. The profit function of an incumbent duopolist is graphed over the remainder of the state space. If an incumbent duopolist has a higher (lower) quality product than its rival, we refer to it as the leader (follower). In Figure 2, the profit function is relatively flat for the follower $\left(\omega_{1}<\omega_{2}\right)$ and relatively steep for the leader $\left(\omega_{1}>\omega_{2}\right)$; i.e., while a follower can increase its profit relatively little by increasing its product quality, a leader can increase its profit significantly by increasing its product quality 7 This stems from the fact that firms face the Logit demand function (2) and, accordingly, an increase in the leader's product quality enhances its demand more than an increase in the follower's product quality enhances its demand (until decreasing returns to quality set in).

\footnotetext{
${ }^{7} \mathrm{~A}$ firm's profit function flattens out as its quality exceeds $\omega^{*}=12$ because of the decreasing returns to quality that set in.
} 

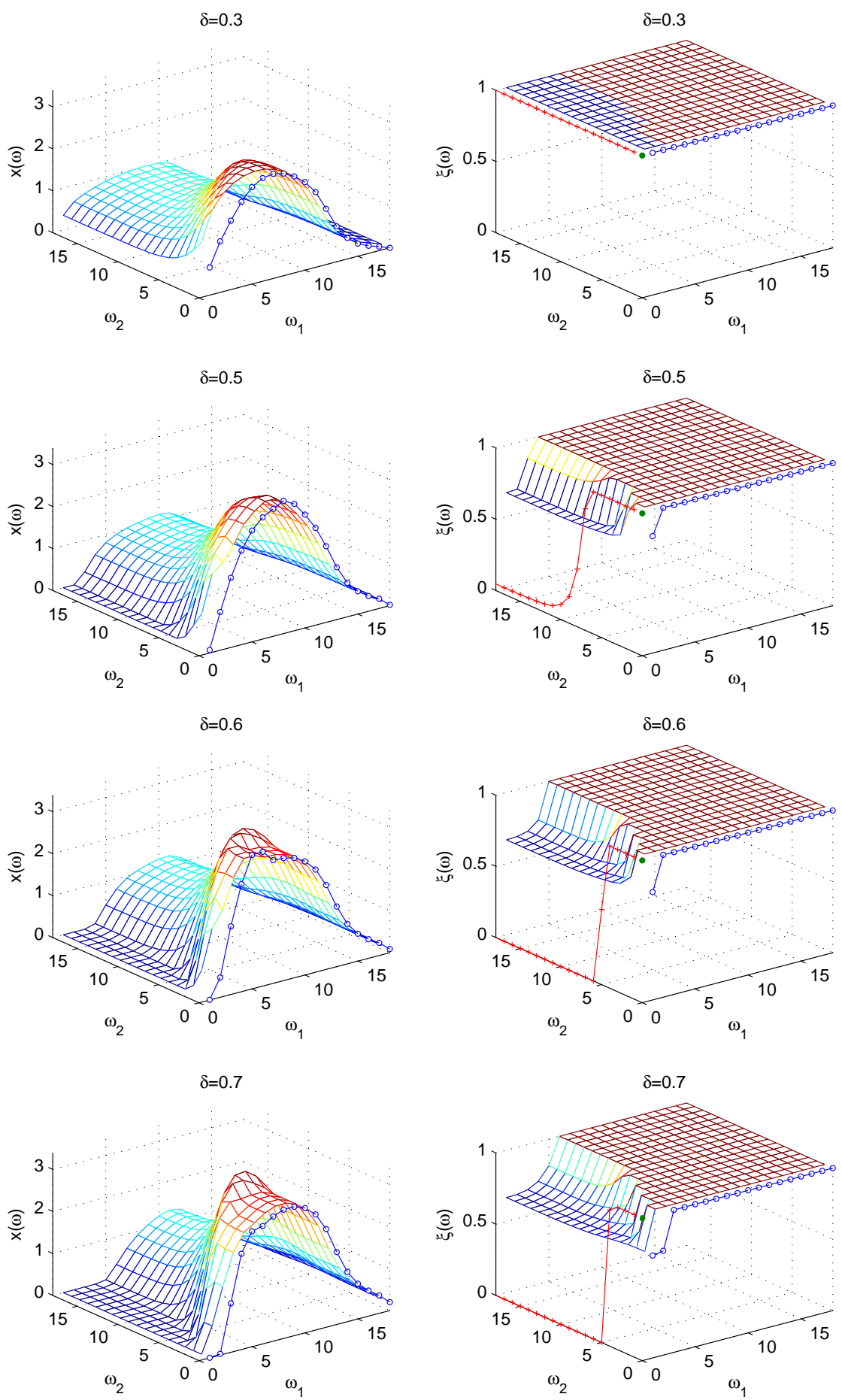

Figure 3: Policy functions $x(\omega)$ (left column) and $\xi(\omega)$ (right column). (Blue = incumbent monopolist; red $=$ potential entrant; green $=$ two potential entrants.) 

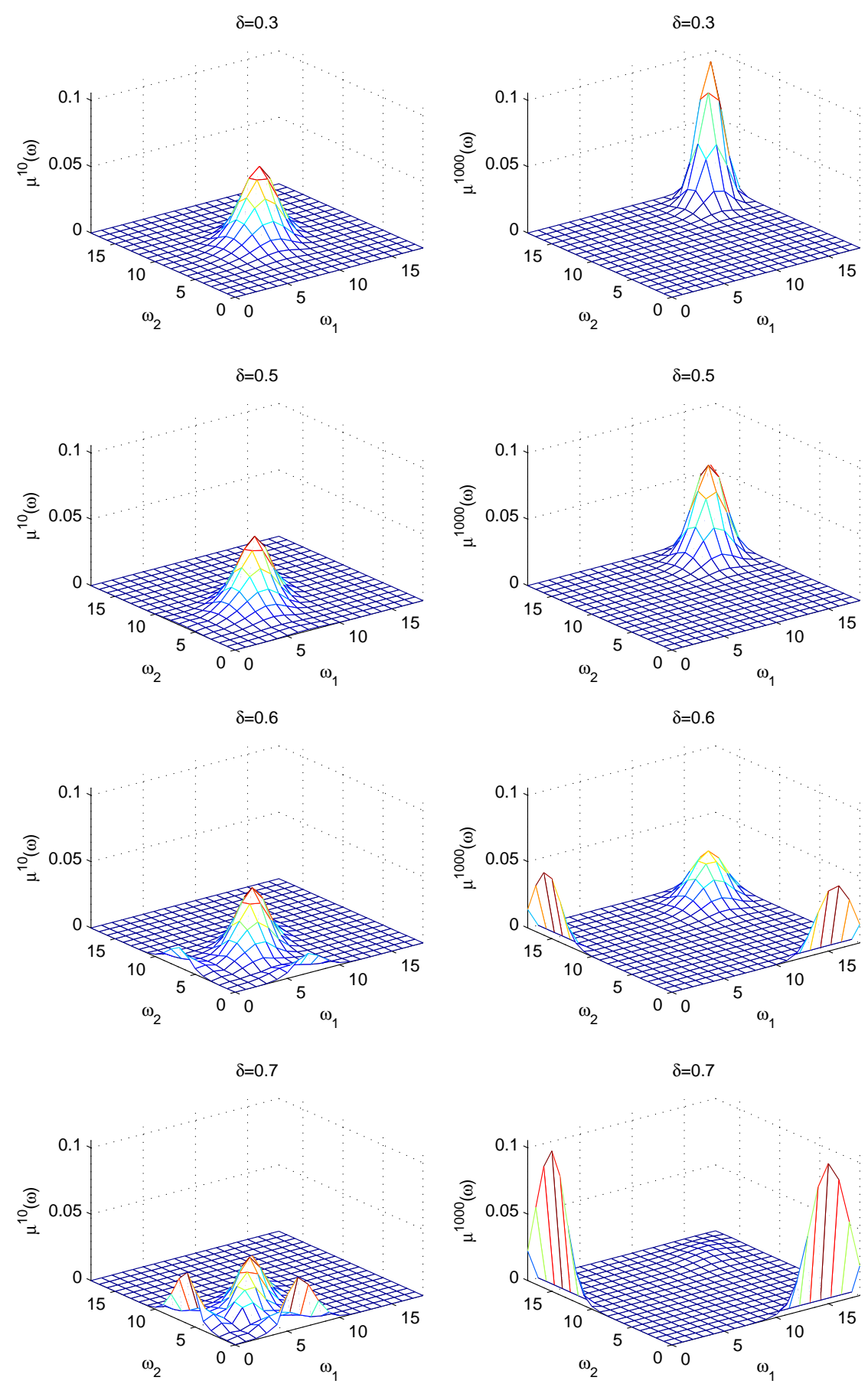

Figure 4: Transient distributions over states in periods 10 (left column) and 1000 (right column) given initial state $(4,4)$. 
Product quality is costly in the sense that an incumbent firm must invest in order to maintain or enhance it. One parameter that affects this cost is the rate of depreciation $\delta$. An increase in the depreciation rate makes it more costly for an incumbent firm to maintain or increase its product quality; i.e., an incumbent firm needs to invest more in order to offset the higher rate at which its product quality decreases. It follows that a higher depreciation rate makes it more costly for a follwer to catch up to or overtake a leader.

To see how the benefits and costs of product quality affect equilibrium behavior and industry dynamics, we present equilibria for various values of $\delta$ - in particular $\delta \in\{0.3,0.5,0.6 ., 0.7\}$. The equilibrium investment and entry/exit policy functions are graphed in Figure 3 in the left and right columns, respectively. The investment and exit policy functions of an incumbent monopolist are graphed in blue along the $\omega_{1}$ axis. The entry policy function of a potential entrant facing an incumbent monopolist is graphed in red along the $\omega_{2}$ axis. The entry policy function of a potential entrant facing an empty industry is graphed in green over state $(0,0)$. The surfaces graphed over the remainder of the state space are the investment and exit policy functions of an incumbent duopolist.

For higher rates of depreciation, a follower that falls sufficiently far behind ceases to invest and exits with positive probability; this can be seen in the subsets of the state space that lie along the $\omega_{2}$ axis in the policy functions for $\delta \in\{0.5,0.6,0.7\}$. A follower in this subset of the state space faces little incentive to invest in order to increase its profit in the imminent future because the profit function is quite flat in this subset (see Figure 2). Furthermore, the follower determines that it is too costly to invest in catching up with the leader, given the high rate of depreciation. Not surprisingly, the higher the rate of depreciation, the larger the subset in which the follower ceases to invest and exits with positive probability; in other words, the higher the depreciation rate, the smaller the lead required to induce the follower to give up. The subset of the state space in which the follower ceases to invest does not necessarily coincide with the subset of the state space in which it exits with positive probability; this depends on the parameterization. Generally speaking, increasing the rate of depreciation causes both of these subsets to grow as they do in Figure 3. So, for a sufficiently high rate of depreciation, an incumbent firm that falls sufficiently far behind both ceases to invest and exits with positive probability.

The leader exploits these incentives by striving to move the industry state into the subset of the state space in which the follower gives up. This can be seen in the policy functions for $\delta \in\{0.6,0.7\}$; the leader invests heavily in the states adjacent to the subset in which the follower gives up. Once in this subset, the leader best responds to its rivals zero investment and imminent exit by significantly decreasing its investment.

We also explore the implications of the equilibrium behavior for industry dynamics, both in the short run and in the long run. We compute the transient distribution over states in period $t, \mu^{t}(\cdot)$, starting from state $\left(\omega^{e}, \omega^{e}\right)=(4,4)$ in period 0 . This tells us how likely each possible industry structure is in period $t$, given that both firms began with the 
exogenous initial product quality. Figures 4 displays the transient distributions in periods 10 and 1000, respectively. We use a transient distribution in period 1000 - instead of an ergodic distribution - to reflect the long-run industry structure because there may be several closed communicating classes 8 When $\delta=0.5$, a follower that falls sufficiently far behind ceases to invest and exits; however, the long-run industry structure is nevertheless symmetric. This is because an incumbent that exits is ultimately replaced by a potential entrant. The transient distributions in Figure 4 show that when the rate of depreciation is sufficiently high, the industry structure will ultimately become asymmetric; the incumbent firm that becomes the leader is very likely to induce its rival to stop investing and exit and thus it ultimately becomes an incumbent monopolist.

Varying other parameters of the model causes equilibrium behavior to change in a similar way - in particular, decreasing the effectiveness of investment, decreasing the market size, or increasing the marginal cost of production 9 More generally, we find that a change in parameterization that increases (decreases) the cost (benefit) of achieving or maintaining any given product quality affects behavior in the same manner as an increase in the rate of depreciation and yields more asymmetric industry structures in the short and long run.

\section{$5 \quad$ Predatory and Limit Investment}

In this section, we explore the effects of entry and exit on equilibrium investment behavior in more detail. In particular, we discuss predatory and limit investment. Predatory and limit investment are most pronounced when an incumbent firm has an incentive to induce exit and prevent entry, respectively. This behavior is less apparent (but present) in the equilibria presented in the previous section because a follower that falls sufficiently far behind is priced out of the market. We can see this by comparing the profit function of a monopolist to the profit function of a duopolist facing a rival in state 1 ; the maximum absolute difference between the functions is 0.028 (for quality levels 13-18), and the maximum relative difference is $0.66 \%$ (for quality level 1). Therefore, to an incumbent, it makes little difference whether it is facing a potential entrant or an incumbent firm with a very low quality product.

To get an unobstructed view of predatory and limit investment, we explore a different parameterization of the product market game - one that gives the follower a higher equilibrium market share in each state, so that it is not priced out of the market. We simply increase the vertical intercept and decrease the slope of the function that maps product

\footnotetext{
${ }^{8} \mathrm{~A}$ closed communicating class is a subset of states that the industry never leaves once it has entered it. When there are multiple closed communicating classes, one cannot compute a single ergodic distribution; rather, one must compute a separate ergodic distribution for each closed communicating class. The transient distribution that we compute instead accounts for the probability of reaching any one of the closed communicating classes. In addition, given a discount factor of $\beta=0.925$ we take a period to be one year; therefore, anything that happens beyond a certain point in time may be considered economically irrelevant.

${ }^{9}$ Although we have not explored the effects of changing period game parameters - such as the market size and the marginal cost of production - on equilibrium behavior using the homotopy method (see equation [30), we have done so using the Pakes \& McGuire (1994) algorithm.
} 
quality into the consumer's valuation of it by replacing $g(\cdot)$ as defined in (1) with

$$
g\left(\omega_{n}\right)=\left\{\begin{array}{ccc}
-\infty & \text { if } & \omega_{n}=0 \\
6+\frac{1}{2} \omega_{n} & \text { if } & 1 \leq \omega_{n} \leq \omega^{*}, \\
6+\frac{1}{2} \omega^{*}+\ln \left(2-\exp \left(\omega^{*}-\omega_{n}\right)\right) & \text { if } & \omega^{*}<\omega_{n} \leq M .
\end{array}\right.
$$

For this parameterization, the difference between the profit function of a monopolist facing a potential entrant and the profit function of a duopolist facing an incumbent firm in state 1 varies from 1.331 (in state 1) to 3.701 (in state 18) in absolute terms and from $17.10 \%$ (in state 18 ) to $53.25 \%$ (in state 1) in relative terms. It follows that an incumbent firm has a very strong incentive to become a monopolist as opposed to a duopolist, no matter how dominant a duopolist it can be. Having given the follower a higher equilibrium market share, we must also increase the setup costs and scrap values; otherwise, incumbent firms would never exit and potential entrants would always enter.

Predatory investment. We assess whether firms engage in predatory investment using the definition inspired by Ordover \& Willig (1981). According to their definition, an action is predatory if it is optimal when taking into consideration its effect on the likelihood that a rival exits, but suboptimal otherwise. Therefore, predation can be studied by comparing firms' policies in two scenarios: in the baseline scenario, scrap values are set to moderate values so that exit is possible but not certain and setup costs are set to values high enough to ensure that entry never occurs $\left(\bar{\phi}=20, \bar{\phi}^{e}=\infty\right)$; the counterfactual scenario differs from the baseline scenario only in that the scrap values are set to such low values that the incumbents never exit $\left(\bar{\phi}=-3, \bar{\phi}^{e}=\infty\right)$. Policy functions for the baseline scenario are presented in the top row of Figure 5. Policy functions for the counterfactual scenario are presented in the middle row of Figure 5. By comparing the investment policy functions, we see exactly how the opportunity to induce exit and become a perpetual monopolist affects an incumbent duopolist's investment incentives. In the bottom row of Figure 5 , we present the difference between the investment policy functions for the two scenarios.

In the difference between investment policy functions, we see a pronounced ridge that is adjacent to the subset of the state space in which the follower ceases investing and exits with positive probability. This demonstrates that in the baseline scenario, a firm invests significantly more than in the counterfactual scenario once it gains a small lead and is in a position to induce its rival to give up. According to the above definition, this additional investment is predatory.

In this model, predation occurs in a complete information setting amongst ex-ante symmetric firms. In the earliest papers in this literature, equilibrium predation was driven by asymmetric information and asymmetries amongst firms (Milgrom \& Roberts 1982, Selten 1978). We contribute to the later stream of the literature that shows that equilibrium predation can occur in the presence of complete information and/or symmetric firms (Cabral 

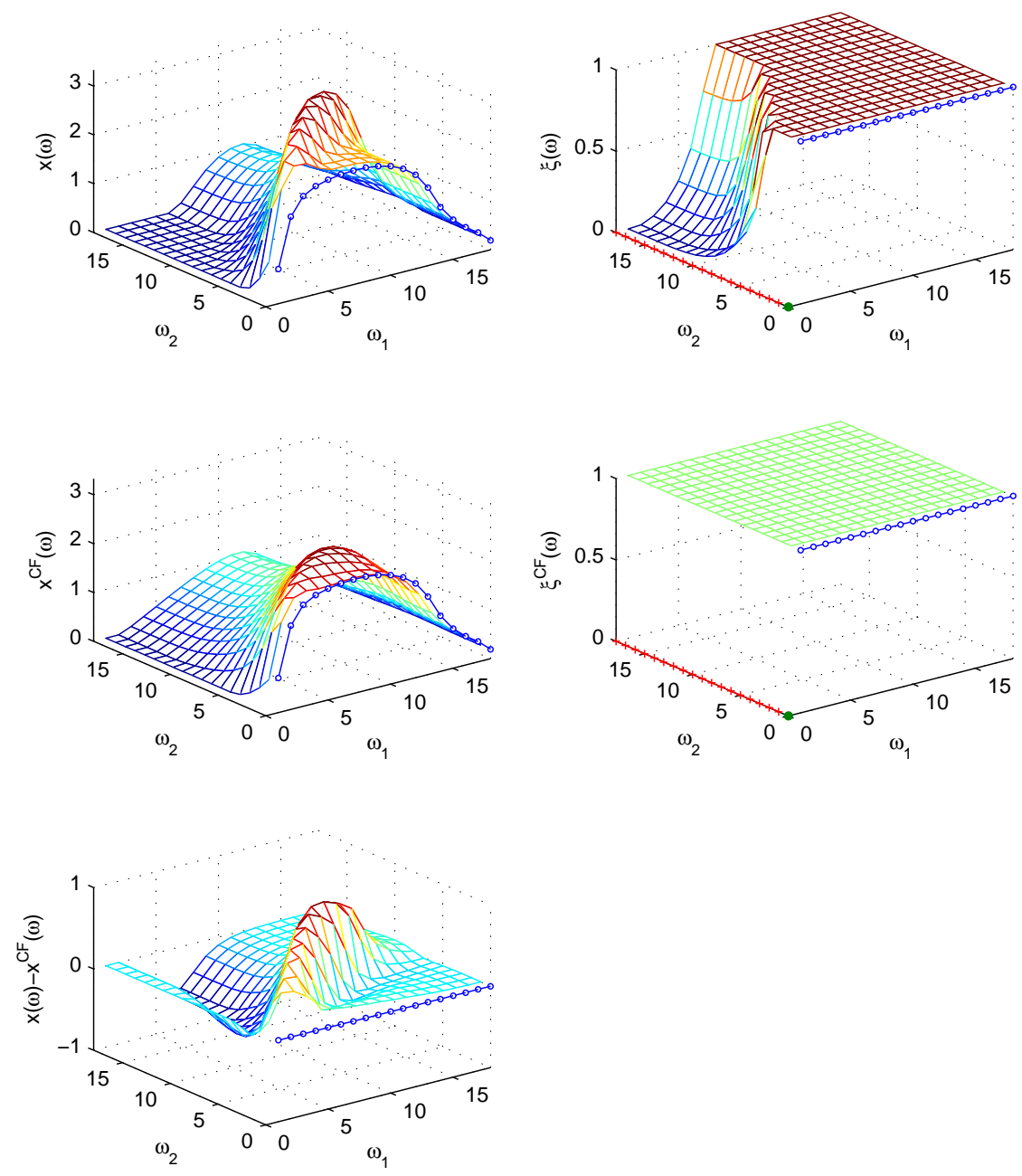

Figure 5: Predatory investment. Baseline scenario policy functions $x(\omega)$ (left column) and $\xi(\omega)$ (right column) for $\bar{\phi}=20$ and $\bar{\phi}^{e}=\infty$ (top row). Counterfactual scenario policy functions $x^{C F}(\omega)$ and $\xi^{C F}(\omega)$ for $\bar{\phi}=-3$ and $\bar{\phi}^{e}=\infty$ (middle row). Difference between investment policy functions (bottom row).

\& Riordan 1994, Cabral \& Riordan 1997, Snider 2008).

Limit investment. We adopt a definition of limit investment analogous to the definition of predatory investment that Ordover \& Willig (1981) inspire; i.e., limit investment occurs when a firm that is threatened with entry invests more than it would have had it not been threatened with entry. In order to assess whether an incumbent monopolist engages in limit investment, we compare two scenarios: the baseline scenario includes setup costs from an intermediate range that induce a potential entrant to enter in only some states, and scrap values set to such low values that incumbents never exit $\left(\bar{\phi}=-3, \bar{\phi}^{e}=22\right)$; the counterfactual scenario differs from the baseline scenario only in that it includes prohibitively high setup costs that induce the potential entrant to refrain from entering in all states $(\bar{\phi}=-3$, $\left.\bar{\phi}^{e}=\infty\right)$. By comparing these scenarios, we see exactly how the opportunity to prevent en- 

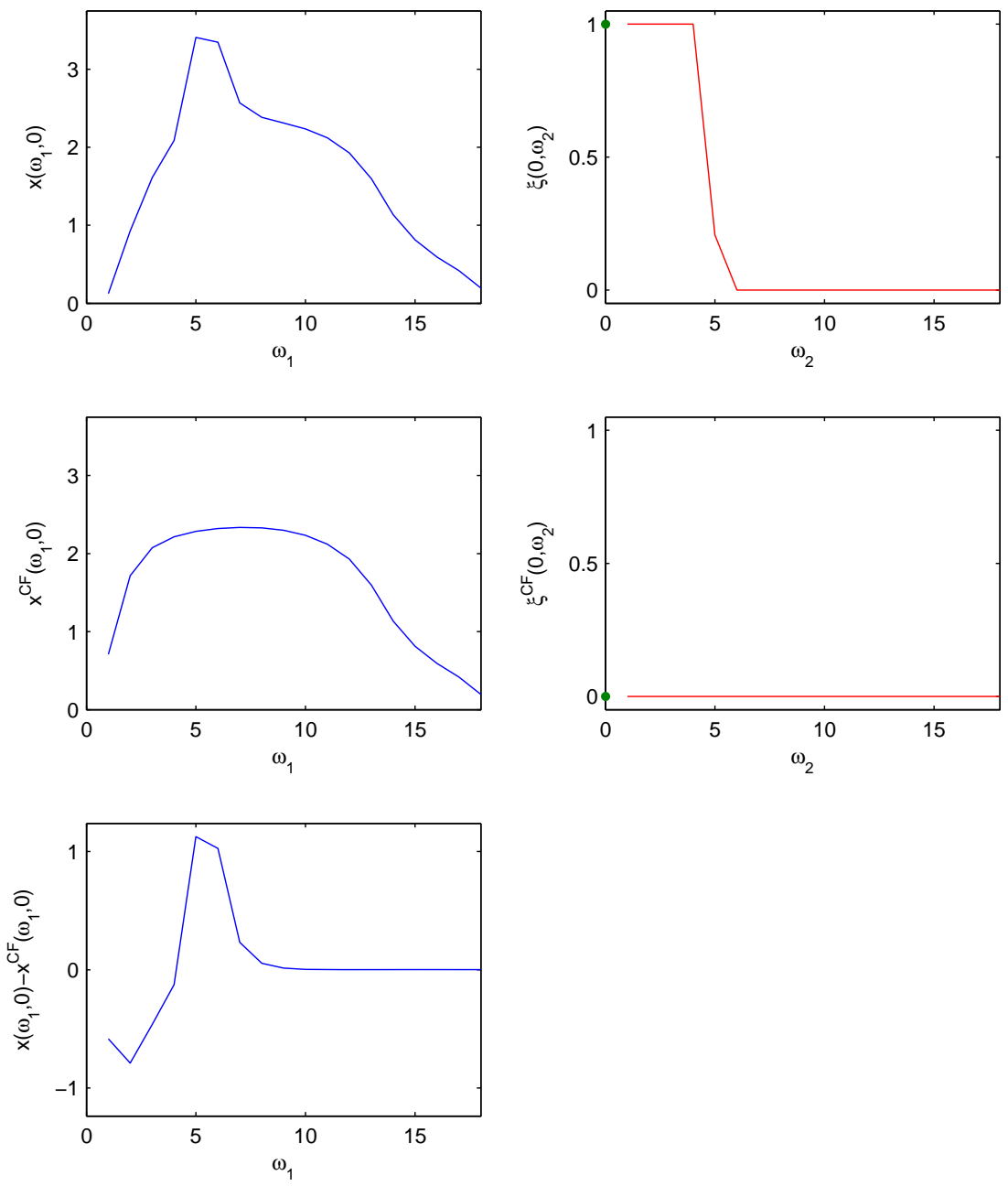

Figure 6: Limit investment. Baseline scenario policy functions $x\left(\omega_{1}, 0\right)$ (left column) and $\xi\left(0, \omega_{2}\right)$ (right column) for $\bar{\phi}=-3$ and $\bar{\phi}^{e}=22$ (top row). Counterfactual scenario policy functions $x^{C F}\left(\omega_{1}, 0\right)$ and $\xi^{C F}\left(0, \omega_{2}\right)$ for $\bar{\phi}=-3$ and $\bar{\phi}^{e}=\infty$ (middle row). Difference between investment policy functions (bottom row).

try and thus prevent the industry from becoming a perpetual duopoly affects an incumbent monopolist's investment incentives. The incumbent monopolist's investment policy function and the potential entrant's entry policy function for the baseline scenario are presented in the top row of Figure 6. The analogous policy functions for the counterfactual scenario are presented in the middle row of Figure 6. By comparing the incumbent monopolist's investment policy functions, we see exactly how the opportunity to prevent entry affects its investment incentives. In the bottom row of Figure 6, we present the difference between the investment policy functions for the two scenarios. As Figure 6 shows, in the baseline scenario, the potential entrant enters if the incumbent monopolist's quality is sufficiently low $\left(\omega_{2} \leq 5\right)$ and does not enter otherwise. Limit investment can be seen in state (5) and neighbouring states, where the incumbent monopolist significantly increases its investment, 

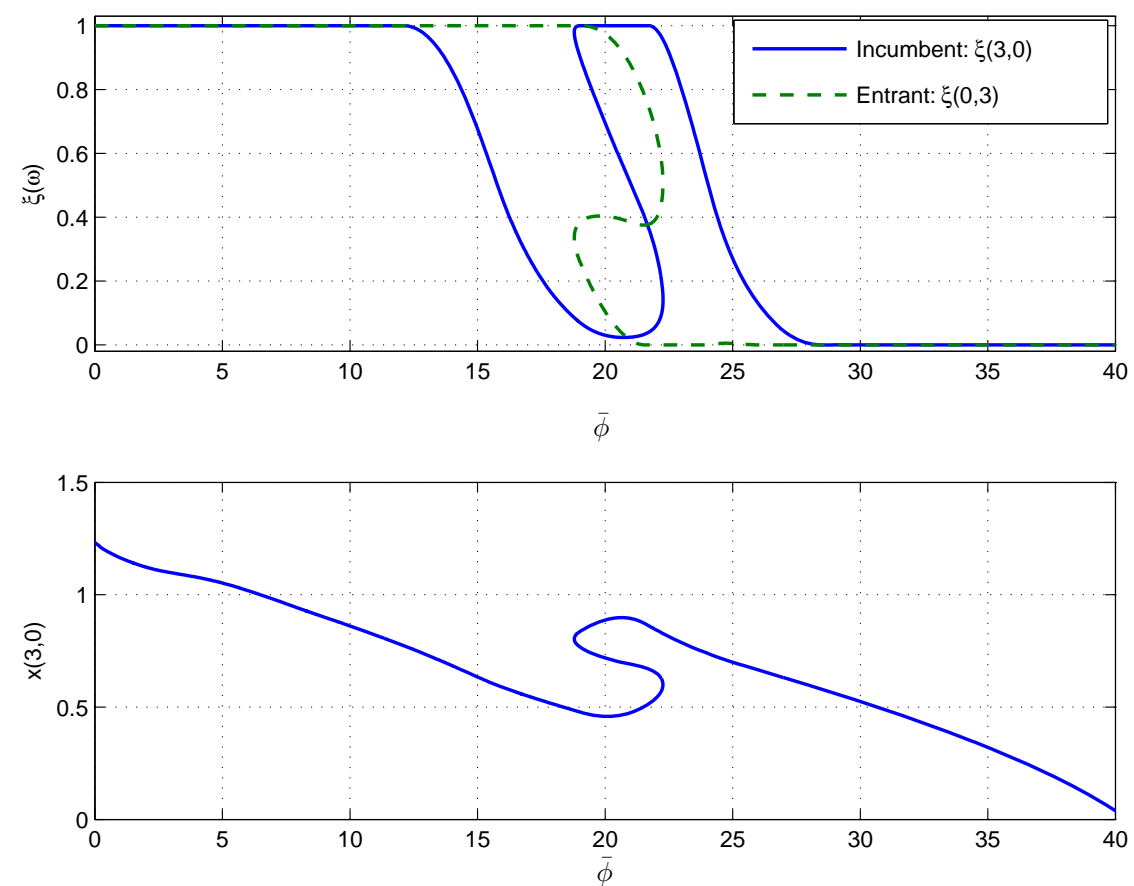

Figure 7: Policy functions for state (3,0): entry/exit policy function (top panel); incumbent firm's investment policy function (bottom panel).

realizing that an increase in its quality will prevent the entrant from entering.

The Pakes \& McGuire (1994) quality ladder model is perhaps the simplest model that one could devise in the Ericson \& Pakes (1995) framework. The fact that introducing entry and exit into this model gives rise to predatory and limit investment therefore suggests that such behaviors are quite pervasive in this framework.

\section{Multiple Equilibria}

Pakes \& McGuire (1994) do not find multiple equilibria of their quality ladder model; on the basis of this, they reason that their quality ladder model does not admit multiple equilibria (p. 570). However, in systematically exploring the equilibrium correspondence of the above model using the homotopy method, we have uncovered several instances of multiplicity. We have searched for multiplicity by allowing the homotopy algorithm to vary several different parameters; see equation (30). While all these searches have uncovered instances of multiplicity, we focus on one specific case. We allow the homotopy algorithm to vary $\bar{\phi}$ and $\bar{\phi}^{e}$; for the baseline parameterization in Table 1, the algorithm traces out a path of equilibria for $\bar{\phi}^{e} \in[0,40]$ and $\bar{\phi}=\bar{\phi}^{e}+2$.

In Figure 7, we show the firms' policy functions for state $(3,0)$, where the differences between equilibria are most prominent. Recall that when the incumbent firm is in state $(3,0)$, the potential entrant is in state $(0,3)$; therefore, we graph the policy functions in 


\begin{tabular}{l|ccc} 
Expected \# of & MPE A & MPE B & MPE C \\
\hline Entering firms & 0.055 & 0.034 & 0.016 \\
Exiting firms & 0.068 & 0.047 & 0.030 \\
Active firms & 1.126 & 1.142 & 1.138
\end{tabular}

Table 2: Summary statistics for period 10 given initial state $(4,4)$.

both of these states. These graphs show that the homotopy algorithm traces out a path that bends back on itself, just as in the example in Figure 1, because the path includes an S-shape in the interval $[18.8,22.3]$. There are multiple equilibria for each parameterization in this interval.

We will explore the three equilibria that arise at $\phi=20$ in more detail. (We do not plot the policy functions for these equilibria because they are qualitatively similar to policy functions presented earlier, in the bottom row of Figure 3.) All three equilibria lead to the same asymmetric (monopolistic) long-run industry structure; for each, the modal states are $(15,0)$ and $(0,15)$. This is not surprising: First, we have already seen that a qualitatively similar equilibrium yields a very asymmetric long-run industry structure (see the bottom row of Figure 4). Second, the policy functions of these three equilibria are virtually identical near the modal states of the long-run industry structure, so we should not expect differences to arise in the long run. However, due to the differences between the policy functions near the origin and along the diagonal of the state space, differences in the short-run industry structures do arise, as demonstrated by the summary statistics in Table 210 For each of the three equilibria, Table 2 presents the expected number of entering, exiting, and active firms in period 10, given that the industry starts from state $\left(\omega^{e}, \omega^{e}\right)=(4,4)$ in period 0 . While there is relatively little variation in the expected number of active firms across the equilibria, there is greater variation in the expected number of entering and exiting firms; both are highest for equilibrium number 1 and lowest for equilibrium number 3 . That is, there is a variation in churn across the equilibria; in equilibrium number 1 (3), firms enter and exit more (less) often in the short run than in the other two equilibria.

Naturally, this churn is directly related to differences in entry and exit probabilities. In the upper panel of Figure 7 , we can see these probabilities for state $(3,0)$. We see that a higher chance of the incumbent firm remaining in the industry is matched by a lower entry probability of the potential entrant, and vice versa: in equilibrium A, the incumbent firm remains in the industry with probability 0.0303 and the potential entrant enters with probability 0.9748 ; in equilibrium B, the incumbent firm remains in the industry with probability 0.6936 and the potential entrant enters with probability 0.4031 ; and in equilibrium $\mathrm{C}$, the incumbent firm remains in the industry with probability 1 and the potential entrant enters with probability 0.1032 . The incumbent most prefers equilibrium

\footnotetext{
${ }^{10}$ Equilibria A, B, and C arise in that order as the homotopy algorithm traces out the path from $\bar{\phi}=0$ to $\bar{\phi}=40$.
} 
number 3 while the potential entrant most prefers equilibrium number 1 ; in equilibrium number 3 , the incumbent is likely to remain an incumbent monopolist, and in equilibrium number 1, the potential entrant is likely to become an incumbent monopolist. Equilibrium behavior of this nature arises in static normal-form coordination games such as "Battle of the Sexes" (see pp. 18-20 of Fudenberg \& Tirole 1991).

All other instances of multiplicity that we have uncovered are qualitatively similar in that the differences between policy functions are similar to those described above. Despite this, we have found multiple equilibria that lead to slight differences in industry structure that persist in the long run; while one equilibrium leads to an extremely symmetric industry structure in which firms are tied, another leads to a slightly asymmetric industry structure in which one firm leads the other by only one quality level. In sum, while we have found multiplicity in the Pakes \& McGuire (1994) quality ladder model, it is hardly as dramatic as in other models (Besanko et al. 2009, Besanko et al. 2008); the differences in equilibria tend to be small and may matter little in practice.

In a companion paper (see Borkovsky et al. 2008), we use the homotopy method to explore a quality ladder model without entry and exit. Interestingly, in the model with entry and exit, multiple equilibria arise for parameterizations for which we did not find multiple equilibria in the model without entry and exit. This suggests that entry and exit may be a source of multiplicity in the Ericson \& Pakes (1995) framework.

\section{Concluding Remarks}

We conduct the first comprehensive exploration of the equilibrium correspondence of the Pakes \& McGuire (1994) quality ladder model. We uncover a variety of interesting equilibrium behavior and economic phenomena.

We find that the industry structure that arises is determined by the cost and benefit of achieving or maintaining any given quality level. The more costly and/or less beneficial it is to achieve or maintain a given quality level, the more a leader invests in striving to induce the follower to give up; the more quickly the follower does so; and the more asymmetric is the industry structure that arises.

We also find that equilibria in the Pakes \& McGuire (1994) model are characterized by predatory and limit investment. As the Pakes \& McGuire (1994) model is a relatively straightforward application of the Ericson \& Pakes (1995) framework, it is likely that such behaviors arise is other models in this framework as well. It is also notable that predation arises in a complete information setting amongst symmetric firms; in much of the earlier literature (e.g., Milgrom \& Roberts 1982, Fudenberg \& Tirole 1986), predation was driven by asymmetric information and/or asymmetries across firms.

Exploring the equilibrium correspondence using the homotopy method allows us to systematically search for multiple equilibria. We find several instances of multiplicity. Furthermore, we find multiple equilibria for parameterizations of the model for which we did 
not find multiple equilibria in the model without entry and exit, suggesting that entry and exit can be a source of multiplicity in the Ericson \& Pakes (1995) framework.

Besides systematically exploring the equilibrium correspondence, the homotopy method has other uses. First, a so-called artificial homotopy can be used to compute an equilibrium for a particular parameterization; in principle, the resulting algorithm is free of convergence problems (Watson et al. 1997, Borkovsky et al. 2008). All-solutions (artificial) homotopies (McKelvey et al. 2006) have been used to solve for all solutions of static games (Bajari, Hong \& Ryan 2009) and may prove useful for dynamic stochastic games as well. Second, Doraszelski \& Escobar (2009) show that in dynamic stochastic games with a finite number of states and actions, the homotopy method can be used to single out the equilibrium that is likely to be played after a policy intervention. Third, the homotopy method could be used in structural estimation of dynamic stochastic games; if all equilibria of a model can be computed, then one can estimate an equilibrium selection rule along with the primitives of the model (Bajari, Hahn, Hong \& Ridder 2008, Bajari et al. 2009, Grieco 2009).

\section{References}

Aguirregabiria, V. \& Mira, P. (2007), 'Sequential estimation of dynamic discrete games', Econometrica 75(1), 1-54.

Bajari, P., Benkard, L. \& Levin, J. (2007), 'Estimating dynamic models of imperfect competition', Econometrica 75(5), 1331-1370.

Bajari, P., Hahn, J., Hong, H. \& Ridder, G. (2008), A note on semiparametric estimation of finite mixturesof discrete choice models, Working paper, University of Minnesota, Minneapolis.

Bajari, P., Hong, H. \& Ryan, S. (2009), 'Identification and estimation of discrete games of complete information', Econometrica forthcoming.

Besanko, D. \& Doraszelski, U. (2004), 'Capacity dynamics and endogenous asymmetries in firm size', Rand Journal of Economics 35(1), 23-49.

Besanko, D., Doraszelski, U., Kryukov, Y. \& Satterthwaite, M. (2009), 'Learning-by-doing, organizational forgetting, and industry dynamics', Econometrica forthcoming.

Besanko, D., Doraszelski, U., Lu, L. \& Satterthwaite, M. (2008), Capacity investment dynamics in oligopolistic industries, Working paper, Northwestern University, Evanston.

Bischof, C., Khademi, P., Mauer, A. \& Carle, A. (1996), 'ADIFOR 2.0: Automatic differentiation of Fortran 77 programs', IEEE Computational Science and Engineering 3(3), 18-32.

Borkovsky, R. (2008), The timing of version releases in R\&D-intensive industries: A dynamic duopoly model, Working paper, University of Toronto, Toronto.

Borkovsky, R., Doraszelski, U. \& Kryukov, S. (2008), A user's guide to solving dynamic stochastic games using the homotopy method, Working paper, Northwestern University, Evanston. 
Cabral, L. \& Riordan, M. (1994), 'The learning curve, market dominance, and predatory pricing', Econometrica 62(5), 1115-1140.

Cabral, L. \& Riordan, M. (1997), 'The learning curve, predation, antitrust, and welfare', Journal of Industrial Economics 45(2), 155-169.

Caplin, A. \& Nalebuff, B. (1991), 'Aggregation and imperfect competition: On the existence of equilibrium', Econometrica 59(1), 26-59.

Chen, J., Doraszelski, U. \& Harrington, J. (2009), 'Avoiding market dominance: Product compatibility in markets with network effects', Rand Journal of Economics 49(3), 455485.

Doraszelski, U. \& Escobar, J. (2009), A theory of regular markov perfect equilibria in dynamic stochastic games: Genericity, stability, and purification, Working paper, Harvard University, Cambridge.

Doraszelski, U. \& Judd, K. (2008), Avoiding the curse of dimensionality in dynamic stochastic games, Technical working paper no. 304, NBER, Cambridge.

Doraszelski, U. \& Markovich, S. (2007), 'Advertising dynamics and competitive advantage', Rand Journal of Economics 38(3), 1-36.

Doraszelski, U. \& Satterthwaite, M. (2009), Computable Markov-perfect industry dynamics: Existence, purification, and multiplicity, Working paper, Harvard University, Cambridge.

Dubé, J., Hitsch, G. \& Manchanda, P. (2005), 'An empirical model of advertising dynamics', Quantitative Marketing and Economics 3, 107-144.

Ericson, R. \& Pakes, A. (1995), 'Markov-perfect industry dynamics: A framework for empirical work', Review of Economic Studies 62, 53-82.

Farias, V., Saure, D. \& Weintraub, G. (2008), The linear programming approach to solving large scale dynamic stochastic games, Working paper, Columbia University, New York.

Ferris, M., Judd, K. \& Schmedders, K. (2007), Solving dynamic games with Newton's method, Working paper, University of Wisconsin, Madison.

Fudenberg, D. \& Tirole, J. (1986), ‘A “signal-jamming” theory of predation', Rand Journal of Economics 17(3), 366-376.

Fudenberg, D. \& Tirole, J. (1991), Game theory, MIT Press, Cambridge.

Goettler, R. \& Gordon, B. (2009), Competition and innovation in the microprocessor industry: Does AMD spur Intel to innovate more?, Working paper, University of Chicago, Chicago.

Gowrisankaran, G. (1999), 'A dynamic model of endogenous horizontal mergers', Rand Journal of Economics 30(1), 56-83.

Gowrisankaran, G. \& Holmes, T. (2004), 'Mergers and the evolution of industry concentration: Results from the dominant firm model', Rand Journal of Economics 35(3), 561582. 
Grieco, P. (2009), An empirical model of discrete choice games with private and common knowledge unobserved variation, Working paper, Northwestern University, Evanston.

Judd, K. (1998), Numerical methods in economics, MIT Press, Cambridge.

Laincz, C. \& Rodrigues, A. (2008), The impact of cost-reducing spillovers on the ergodic distribution of market structures, Working paper, Drexel University, Philadelphia.

Langohr, P. (2004), Competitive convergence and divergence: Position and capability dynamics, Working paper, Bureau of Labor Statistics, Washington, DC.

Markovich, S. (2008), 'Snowball: A dynamic oligopoly model with network externalities', Journal of Economic Dynamics and Control 32, 909-938.

Markovich, S. \& Moenius, J. (2009), 'Winning while losing: Competition dynamics in the presence of indirect network effects', International Journal of Industrial Organization forthcoming.

McKelvey, R., McLennan, A. \& Turocy, T. (2006), Gambit: Software tools for game theory, Technical report, California Institute of Technology, Pasadena.

Milgrom, P. \& Roberts, J. (1982), 'Predation, reputation, and entry deterrence', Journal of Economic Theory 27, 280-312.

Narajabad, B. \& Watson, R. (2008), The dynamics of innovation and horizontal differentiation, Working paper, University of Texas at Austin, Austin.

Ordover, J. \& Willig, R. (1981), 'An economic definition of predation: Pricing and product innovation', Yale Law Journal 91, 8-53.

Pakes, A. \& McGuire, P. (1994), 'Computing Markov-perfect Nash equilibria: Numerical implications of a dynamic differentiated product model', Rand Journal of Economics 25(4), 555-589.

Pakes, A. \& McGuire, P. (2001), 'Stochastic algorithms, symmetric Markov perfect equilibrium, and the "curse" of dimensionality', Econometrica 69(5), 1261-1281.

Pakes, A., Ostrovsky, M. \& Berry, S. (2007), 'Simple estimators for the parameters of discrete dynamic games (with entry/exit examples)', Rand Journal of Economics 38(2), 373-399.

Pesendorfer, M. \& Schmidt-Dengler, P. (2008), 'Asymptotic least squares estimators for dynamic games', Review of Economic Studies 75(3), 901-928.

Santos, C. (2009), Solving dynamic games by discretizing the state distribution, Working paper, University of Alicante, Alicante.

Selten, R. (1978), 'The chain-store paradox', Theory and Decision 9(2), 127-159.

Snider, C. (2008), Predatory incentives and predation policy: the American Airlines case, Working paper, University of Minnesota, Minneapolis.

Song, M. (2008), A dynamic analysis of cooperative research in the semiconductor industry, Working paper, University of Rochester, Rochester. 
Verschelde, J. (1999), 'Algorithm 795: PHCpack: a general-purpose solver for polynomial systems by homotopy continuation', ACM Transcations on Mathematical Software 25(2), 251-276.

Watson, L., Billups, S. \& Morgan, A. (1987), 'HOMPACK: A suite of codes for globally convergent homotopy algorithms', ACM Transcations on Mathematical Software 13(3), 281-310.

Watson, L., Sosonkina, M., Melville, R., Morgan, A. \& Walker, H. (1997), 'Algorithm 777: HOMPACK90: A suite of Fortran 90 codes for globally convergent homotopy algorithms', ACM Transcations on Mathematical Software 23(4), 514-549.

Weintraub, G., Benkard, L. \& Van Roy, B. (2008), Computational methods for oblivious equilibrium, Working paper, Columbia University, New York.

Zangwill, W. \& Garcia, C. (1981), Pathways to solutions, fixed points, and equilibria, Prentice Hall, Englewood Cliffs. 